\title{
Testing gravity with halo density profiles observed through gravitational lensing
}

\author{
Tatsuya Narikawa and Kazuhiro Yamamoto \\ Department of Physical Science, Hiroshima University, Higashi-Hiroshima 739-8526, Japan \\ E-mail: narikawa@theo.phys.sci.hiroshima-u.ac.jp, kazuhiro@hiroshima-u.ac.jp
}

\begin{abstract}
We present a new test of the modified gravity endowed with the Vainshtein mechanism with the density profile of a galaxy cluster halo observed through gravitational lensing. A scalar degree of freedom in the galileon modified gravity is screened by the Vainshtein mechanism to recover Newtonian gravity in high-density regions, however it might not be completely hidden on the outer side of a cluster of galaxies. Then the modified gravity might yield an observational signature in a surface mass density of a cluster of galaxies measured through gravitational lensing, since the scalar field could contribute to the lensing potential. We investigate how the transition in the Vainshtein mechanism affects the surface mass density observed through gravitational lensing, assuming that the density profile of a cluster of galaxies follows the original Navarro-Frenk-White (NFW) profile, the generalized NFW profile and the Einasto profile. We compare the theoretical predictions with observational results of the surface mass density reported recently by other researchers. We obtain constraints on the amplitude and the typical scale of the transition in the Vainshtein mechanism in a subclass of the generalized galileon model.
\end{abstract}

Keywords: modified gravity, gravitational lensing 


\section{Contents}

1 Introduction $\quad 1$

2 Formulation $\quad 3$

2.1 Modified gravity model 3

2.2 Gravitational lensing in modified gravity 5

2.3 Parametrization of the modified gravity 6

3 Halo density profiles and differential surface mass density 4

3.1 NFW profile $\quad 9$

$\begin{array}{lll}3.2 & \text { gNFW profile } & 12\end{array}$

$\begin{array}{lll}3.3 & \text { Einasto profile } & 13\end{array}$

$\begin{array}{lll}3.4 & \text { Differential surface mass density } & 15\end{array}$

4 Results - Comparison with observations - 16

$\begin{array}{llr}5 & \text { Discussion } & 18\end{array}$

6 Summary and Conclusion $\quad 20$

A The coefficients in the perturbation equations $\quad 22$

B Galileon model $\quad 23$

\section{Introduction}

Discovery of the late-time accelerated expansion of our universe [1, 2] boosted interests in nature of gravity on cosmological scales, which might be a key to explore its origin (e.g., Refs. [3-5]). Modification of gravity is an alternative approach to dark energy paradigm to explain the cosmic accelerated expansion [3-7]. There have been proposed, for example, $f(R)$ model [8-12], Dvali-Gabadadze-Porrati (DGP) model [13], and the galileon model [14]. In general, it is very challenging to construct a viable model that explains the late-time accelerated expansion of the background universe, satisfying the local gravity constraints without theoretical plague simultaneously.

The DGP model is described in the context of the braneworld scenario, which consists of a 3+1-dimensional brane embedded in a 5-dimensional bulk. This model has an interesting phenomenology, which yields two branches of the Friedmann equation, a self-accelerating branch (sDGP) and a normal branch (nDGP). In the sDGP branch, the expansion of the background universe self-accelerates at late times without a cosmological constant, while the nDGP branch needs to add a stress-energy component with negative pressure on the brane to be consistent with cosmological observations $[15,16]$. Unfortunately, the sDGP model inevitably gives rise to a ghost $[17,18]$. Moreover, the sDGP model is disfavored by the cosmological observations [19-21].

Inspired by the decoupling limit of the DGP model [17, 22], the galileon gravity theory has been studied as a possible alternative to large distance modification of gravity (e.g., [14, 23-33]). This theory introduces a scalar field invariant under the Galilean shift symmetry 
$\partial_{\mu} \phi \rightarrow \partial_{\mu} \phi+b_{\mu}$ in the Minkowski space-time, which keeps the equation of motion being the second order differential equation. Although the Lagrangian no longer satisfies the Galilean shift symmetry in a curved spacetime, one can construct the generalized galileon model whose field equation remains a second order differential equation, which simultaneously admits the self-accelerating solution in a FRW universe without a ghost instability [34-36].

In general, the gravitational force and the gravitational potential are modified on the scales of cosmology as a result of the modification of gravity. However, the solar system observations require that Newtonian gravity is recovered on those scales [37]. There have been proposed a few successful screening mechanisms depending on modified gravity models, e.g., the chameleon mechanism [38-40], the symmetron [41, 42], and the Vainshtein mechanism [43]. One of the notable features of the galileon gravity is the Vainshtein mechanism, which hides the scalar field to recover Newtonian gravity in high-density regions. In the Vainshtein mechanism, the self-interaction term like $(\partial \phi)^{2} \square \phi$ plays an important roll. Due to the nonlinear interaction, the scalar field is suppressed in high-density regions, hence it is screened there. Thanks to this screening mechanism, the galileon gravity models can evade the solar-system constraints [14] (c.f. [44]).

It is worth examining whether the screening mechanism works completely or not around high-density regions on cosmological scales. The structure of halos of galaxy and cluster of galaxies may be useful to test these modified gravity models. Recently, several researchers have investigated such a possibility of testing these modified gravity models on the scales of halos of galaxy and cluster of galaxies [45-57]. In [58], the authors have investigated a constraint on the Vainshtein mechanism in the context of the massive gravity, using gravitational lensing and velocity dispersion data from galaxies. In a recent work, we investigated how the transition in the Vainshtein mechanism may appear in the sDGP model and the galileon model [54]. We found that the circular speed of a test particle in the sDGP and the galileon model deviates from that in Newtonian gravity at $10 \%$ level on the outer region of a halo with the Navarro-Frenk-White (NFW) density profile (e.g., [59,60]). Thus the Vainshtein mechanism might not completely hide the effect of the modified gravity on the cluster's scales.

The purpose of the present paper is to investigate how the transition in the Vainshtein mechanism in a subclass of the generalized galileon model appears in an observation of a halo density profile measured through the gravitational lensing phenomena. We consider a subclass of the most general second-order scalar-tensor theory [34-36, 61], in which the scalar field modifies the equation connecting the lensing potential and the matter density. In this model, the observational quantities measured through the lensing phenomena are contaminated by the scalar field's effect, which might leave testable signatures in observed halos. Then, we focus our investigation on the signature of the modified gravity by comparing theoretical predictions and observations.

Many works have been done for testing gravity on cosmological scales using, for example, the large scale structures of galaxies and the redshift-space distortions (e.g., [62-67]), and the integrated Sachs-Wolfe effect through cross-correlations between the cosmic microwave background temperature anisotropies and the galaxy distributions (e.g., [68]). The cluster abundance (e.g., [69]) provides a test of gravity on rather smaller scales, but it reflects the regime of the linear evolution of density perturbations substantially. On smaller scales, other tests of deviation from general relativity have been done $[70-74,78]$. A halo of cluster of galaxies provides a unique chance to test the gravity theory on the scale between the solarsystem and the large scale structure of galaxies, where the nonlinear effect of the density 
perturbations plays an important role.

In the present paper, we focus our investigation on the surface mass density of cluster halo measured with gravitational lensing. Cluster lensing surveys are in progress, which is useful to obtain mass distribution of clusters over a wide range of radius by combing strong and weak lensing data. Recently, measurements of the surface mass density were reported using the gravitational lensing [56, 75-77]. The error of the stacked data is small, which is useful to test the modified gravity as will be demonstrated below. Umetsu et al. derived a mean surface mass profile of four clusters, A1689, A1703, A370, and Cl0024+17, from Hubble Space Telescope and Subaru images, in the range $R=(40-2800) h^{-1} \mathrm{kpc}$ at the mean lensing redshift $\left\langle z_{l}\right\rangle=0.32[75,76]$. They obtained mass profiles with highprecision, by combining independent strong-lensing measurements, weak-lensing distortion, and magnification. Oguri et al. presented the mass distribution of a sample of 25 galaxy clusters, in the range $R=(63-5010) h^{-1} \mathrm{kpc}$ at the mean lensing redshift $\left\langle z_{l}\right\rangle=0.47$ and the mean source redshift $\left\langle z_{s}\right\rangle=1.1$ [77]. The cluster sample is based on the Sloan Giant Arcs Survey (SGAS) from the Sloan Digital Sky Survey (SDSS). They derived the differential surface mass density by combining the strong lensing information from the giant arcs and weak lensing measurements from Subaru/Suprime-Cam [79] images. Umetsu et al. and Oguri et al. fit the data within the general relativity, and they found that the NFW profile favors the data.

We confront the observed surface mass density of clusters with the theoretical prediction of the modified gravity model endowed with the Vainshtein mechanism. In the theoretical modeling, we introduce the parameters $\mu$ and $\epsilon$, respectively, which characterize the amplitude and the typical radius of the transition in the Vainshtein mechanism. We obtain constraints on $\mu$ and $\epsilon$. This demonstrates the usefulness of the surface mass density to constrain the modified gravity model.

The structure of this paper is as follows. In section 2, we derive our basic theoretical formulas in a subclass of the generalized galileon modified gravity to compare with observational quantities from gravitational lensing. In section 3, we scrutinize the theoretical behavior of the surface mass density profile depending on familiar density profiles. In section 4 , we present the results of the confrontation between the theoretical predictions and the observational results, which derive constraints on the model parameters $\mu$ and $\epsilon$ characterizing the modification of gravity. In section 5, we discuss about the results and our interpretations. Section 6 is devoted to summary and conclusions. In appendix A, we summarize the definitions of the coefficients in the perturbation equations in section 2. In appendix B, we summarize the coefficients in the perturbation equations in the original galileon model.

Throughout this paper, we use units in which the speed of light equals unity, and we follow the convention $(-,+,+,+)$. We use the reduced Planck mass $M_{\mathrm{Pl}}$, defined by $M_{\mathrm{Pl}}=1 / \sqrt{8 \pi G}$ with Newton's gravitational constant $G$. We adopt the Hubble constant $H_{0}=100 h \mathrm{~km} / \mathrm{s} / \mathrm{Mpc}$ with $h=0.702$, and the matter density parameter at present $\Omega_{0}=$ $0.275[80]$.

\section{Formulation}

\subsection{Modified gravity model}

The most general second-order scalar-tensor theory was derived by Horndeski [34] for the first time, which was recently rediscovered by Deffayet et al. [35] as the most generalized galileon theory, which contains four arbitrary functions of the functions of $\phi$ and $X=$ 
$-g^{\mu \nu} \nabla_{\mu} \phi \nabla_{\nu} \phi / 2$. The Vainshtein mechanism in the generalized galileon theory is discussed recently [44], which clarified variety of the solutions of the scalar field in the generalized theory. We here consider a subclass of the general second-order scalar-tensor theories in a curved spacetime, which is nonminimally coupled to gravity with the action [61],

$$
S=\int d^{4} x \sqrt{-g}\left[\frac{1}{2} F(\phi) R+K(\phi, X)-G(\phi, X) \square \phi+\mathcal{L}_{\mathrm{m}}\right],
$$

where $K(\phi, X)$ and $G(\phi, X)$ are the arbitrary functions of $\phi$ and $X, F(\phi)$ is the function of $\phi$, and $\mathcal{L}_{\mathrm{m}}$ is the matter Lagrangian. We assume that the matter fields do not have direct couplings with the field $\phi$. This corresponds to the Lagrangian with $G_{4}=F(\phi) / 2$ and $G_{5}=0$ in the most general galileon model [36]. The action reduces to the kinetic gravity braiding model, for the choice $F(\phi)=M_{\mathrm{Pl}}^{2}[29,33]$. The original galileon model is reproduced by choosing $F(\phi)=M_{\mathrm{Pl}}^{2}, K(\phi, X)=-X$ and $G(\phi, X)=\left(r_{c}^{2} / M_{\mathrm{Pl}}\right) X$, where $r_{c}$ is the parameter [29].

In this subsection, we summarize the perturbation equations for gravity and the scalar field in the cosmological background. In reference [44], the Vainshtein mechanism in the most general second-order scalar-tensor theory was investigated. The model in the present paper (2.1), a subclass of the most general second-order scalar-tensor theory, was also investigated therein. Following [44], we choose the Newtonian gauge,

$$
d s^{2}=-(1+2 \Psi(t, \mathbf{x})) d t^{2}+a(t)^{2}(1+2 \Phi(t, \mathbf{x})) \delta_{i j} d x^{i} d x^{j},
$$

where $a(t)$ is the scale factor. Within the subhorizon scales with the quasi-static approximation, we have the following perturbed equations,

$$
\begin{aligned}
& \frac{\triangle}{a^{2}} \Phi=-4 \pi G \delta \rho+\xi \frac{\triangle}{a^{2}} \varphi, \\
& \Phi+\Psi=-\alpha \varphi,
\end{aligned}
$$

and

$$
\frac{\triangle}{a^{2}} \varphi+\lambda^{2}\left(\frac{\varphi, i j}{a^{2}} \frac{\varphi^{, i j}}{a^{2}}-\left(\frac{\triangle}{a^{2}} \varphi\right)^{2}\right)=-4 \pi G \zeta \delta \rho,
$$

where $\varphi(\mathbf{x})$ denotes the perturbation of scalar field defined by $\phi(t, \mathbf{x})=\phi(t)(1+\varphi(\mathbf{x}))$, $\triangle$ represents the Laplace differentiation operator, $\delta \rho$ is the perturbed matter density, the Newton's gravitational constant is defined by $G \equiv 1 /(8 \pi F(\phi))$, and the coefficients $\alpha, \xi, \zeta$, and $\lambda^{2}$ depend on the functions $F(\phi), K(\phi, X)$, and $G(\phi, X)$, whose explicit expressions are summarized in appendix A.

The above equations are derived as follows (see [44] for details). We consider the gravitational and scalar fields on subhorizon scales sourced by a non-relativistic matter density perturbation $\delta \rho$. Then, we may ignore time derivative in the field equations, while keeping spatial derivatives. Denoting the quantities for perturbations $\Psi, \Phi$ and $\varphi$ by $\mathcal{V}$, we keep all the terms $\left(\partial^{2} \mathcal{V}\right)^{n}$ with $n \geq 1$, because the $L^{2}(t) \partial^{2} \mathcal{V}$ could be large on small scales, where $L(t)$ is a typical length scale associated with the background evolution which may be as large as the Hubble radius. Within this framework, it has been shown that the terms $\left(\partial^{2} \mathcal{V}\right)^{n}$ with $n \geq 2$ do not appear in the gravitational field equations (2.3) and (2.4) for our model (2.1). But only the terms of $\left(\partial^{2} \mathcal{V}\right)^{2}$ appear in the scalar field equation (2.5). 
Note that the coefficients $\alpha, \xi, \zeta$, and $\lambda^{2}$ are determined by the background field evolution depending on $F(\phi), K(\phi, X)$, and $G(\phi, X)$. The coefficients may take any value because $F(\phi), K(\phi, X)$, and $G(\phi, X)$ are arbitrary functions of $\phi$ and $X$. We should choose $F(\phi)$, $K(\phi, X)$, and $G(\phi, X)$ requiring the accelerated expansion of the universe and satisfying the conditions of no ghost nor instability, which will limit the arbitrary functions. However, we here assume that $\alpha, \xi, \zeta$, and $\lambda^{2}$ may take any value reflecting the arbitrariness of the functions in the model, and consider the constraint from observation through gravitational lensing.

Combining (2.3) and (2.4), we have

$$
\frac{\triangle}{a^{2}}\left(\frac{\Psi-\Phi}{2}\right)=4 \pi G \delta \rho-\frac{\alpha+2 \xi}{2} \frac{\triangle}{a^{2}} \varphi .
$$

In the spherically symmetric case, $(2.5)$ yields

$$
\frac{d \varphi}{d r}=\frac{r}{4 \lambda^{2}}\left(1-\sqrt{1+\frac{8 G \lambda^{2} \zeta M(r)}{r^{3}}}\right),
$$

where we defined the enclosed mass $M(r)=4 \pi \int_{0}^{r} d r^{\prime} r^{\prime 2} \delta \rho\left(r^{\prime}\right)$. In the spherically symmetric case, (2.7) leads to

$$
\frac{\triangle}{a^{2}} \varphi=\frac{1}{r^{2}} \frac{d}{d r}\left(r^{2} \frac{d \varphi}{d r}\right)=\frac{3}{4 \lambda^{2}}\left(1-\sqrt{1+\frac{8 G \lambda^{2} \zeta M(r)}{r^{3}}}\right)-G \zeta \frac{4 \pi r^{3} \delta \rho(r)-3 M(r)}{r^{3} \sqrt{1+\frac{8 G \lambda^{2} \zeta M(r)}{r^{3}}}} .
$$

As we will show in the below (see also [44]), the lensing signature under the influence of the Vainshtein mechanism in our model (2.1) is described by the two combination of the parameters $\lambda^{2} \zeta$ and $(\alpha+2 \xi) \zeta / 2$, on which we focus our investigation. In the original galileon model, we have non-zero values of $\lambda^{2} \zeta$ and $(\alpha+2 \xi) \zeta / 2$ (see appendix B). On the other hand, in the model of the sDGP model, we have $\alpha=-1$ and $\xi=1 / 2$, then $(\alpha+2 \xi) \zeta / 2$ reduces to zero, while $\lambda^{2} \zeta$ takes a non-zero value. ${ }^{1}$ Therefore, in the sDGP model, the relation between the lensing potential $(\Phi-\Psi) / 2$ and the matter density perturbation (2.6) is the same as that in the general relativity. In this case, our method cannot put a constraint because there is no effect of the modified gravity on the gravitational lensing in such a class of models.

In order to obtain the closed system of equations, we need another equation for the matter density perturbation $\delta \rho$. Because we are considering the model in which the matter is minimally coupled with the scalar field, the matter component follows the usual equation under the influence of the gravitational potential $\Psi$. Instead of specifying the coupled equations of $\delta \rho$ and $\Psi$, we simply assume the NFW density profile or other well-known profiles for the matter density perturbation $\delta \rho$ (cf. [54]). From N-body simulations in reference [46], it is suggested that the NFW profile describes the density profile of halos in the DGP model though the parameters of the profiles are scaled. This is a supporting evidence of our assumption in the present paper.

\subsection{Gravitational lensing in modified gravity}

Now let us consider observational quantities observed through gravitational lensing. The light propagation in the Newtonian gauge (2.2) is described by the lensing potential $(\Phi-\Psi) / 2$.

\footnotetext{
${ }^{1}$ In the spatially flat sDGP model, we have $\lambda^{2} \zeta=2\left(r_{c} / 3 \beta\right)^{2}$, where $r_{c}=1 /\left[\left(1-\Omega_{m}\right) H_{0}\right]$ and $\beta=$ $1-2 H r_{c}\left(1+\dot{H} / 3 H^{2}\right)$.
} 
For example, the convergence $\kappa(\chi)$ of the gravitational lensing is given by (e.g., $[81,82])$

$$
\kappa \simeq-\frac{1}{2} \int_{0}^{\chi} d \chi^{\prime} \frac{f_{K}\left(\chi-\chi^{\prime}\right) f_{K}\left(\chi^{\prime}\right)}{f_{K}(\chi)} \triangle^{(2 D)}(\Phi-\Psi),
$$

where $f_{K}(\chi)$ is the comoving angular diameter distance and $\triangle^{(2 D)}$ is the comoving two dimensional Laplacian. Note that $f_{K}(\chi)=\chi$ for the spatially flat universe. The shear can be written in a similar form with (2.9). It is worthy to note that any quantity of gravitational lensing is described by the lensing potential.

Using the thin lens approximation, (2.9) and (2.6) yield

$$
\kappa \simeq \frac{f_{K}\left(\chi_{\mathrm{S}}-\chi_{\mathrm{L}}\right) f_{K}\left(\chi_{\mathrm{L}}\right)}{f_{K}\left(\chi_{\mathrm{S}}\right)} \int_{0}^{\chi_{\mathrm{S}}} d \chi^{\prime}\left[4 \pi G \rho\left(r^{\prime}\right)-\frac{\alpha+2 \xi}{2} \frac{\triangle}{a_{\mathrm{L}}^{2}} \varphi\right] a_{\mathrm{L}}^{2},
$$

where $\chi_{\mathrm{S}}$ is the comoving distance between the observer and the source, $\chi_{\mathrm{L}}$ is the comoving radial distance between the observer and the lens object, and $a_{\mathrm{L}}$ is the scale factor at which we observe the lens object. We define the observed surface mass density, which is obtained through the gravitational lensing phenomena,

$$
\Sigma_{\mathrm{S}}=\Sigma_{\text {crit }} \kappa=\int_{0}^{\chi_{\mathrm{S}}} d\left(a_{\mathrm{L}} \chi^{\prime}\right)\left[\rho\left(r^{\prime}\right)-\frac{\alpha+2 \xi}{8 \pi G} \frac{\triangle}{a_{\mathrm{L}}^{2}} \varphi\right]
$$

where $\Sigma_{\text {crit }}$ is defined by

$$
\Sigma_{\text {crit }}=\frac{1}{4 \pi G} \frac{f_{K}\left(\chi_{\mathrm{S}}\right)}{f_{K}\left(\chi_{\mathrm{L}}\right) f_{K}\left(\chi_{\mathrm{S}}-\chi_{\mathrm{L}}\right) a_{\mathrm{L}}} .
$$

We introduce the spatial coordinate being the physical coordinate, whose origin is located at the center of the lens object by $Z=a_{\mathrm{L}}\left(\chi-\chi_{\mathrm{L}}\right)$ and $r_{\perp}=a_{\mathrm{L}} f_{K}\left(\chi_{\mathrm{L}}\right) \theta$, where $\theta$ is the polar angle of the polar axis connecting the observer and the lens object. Then, using (2.8), the observed surface mass density (2.11) is written as

$$
\begin{array}{r}
\Sigma_{\mathrm{S}}\left(r_{\perp}\right)=2 \int_{0}^{\infty} d Z\left[\rho(r)-\frac{3(\alpha+2 \xi)}{32 \pi G \lambda^{2}}\left(1-\sqrt{1+\frac{8 G \lambda^{2} \zeta M(r)}{r^{3}}}\right)\right. \\
\left.+\frac{(\alpha+2 \xi) \zeta}{8 \pi} \frac{4 \pi r^{3} \rho(r)-3 M(r)}{r^{3} \sqrt{1+\frac{8 G \lambda^{2} \zeta M(r)}{r^{3}}}}\right],
\end{array}
$$

where $r=\sqrt{r_{\perp}^{2}+Z^{2}}$.

\subsection{Parametrization of the modified gravity}

To investigate the constraint on the modified gravity model on the halo scales, we introduce the parameters $\mu$ and $\epsilon$ as follows, which characterize the modification of gravity,

$$
\mu=\frac{(\alpha+2 \xi) \zeta}{2}, \quad \epsilon=\sqrt{H_{0}^{2} \lambda^{2} \zeta}
$$

With these two parameters the surface mass density (2.13) is written as

$$
\Sigma_{\mathrm{S}}\left(r_{\perp}\right)=2 \int_{0}^{\infty} d Z\left[\rho(r)-\frac{3 H_{0}^{2} \mu}{16 \pi G \epsilon^{2}}\left(1-\sqrt{1+\frac{8 G \epsilon^{2} M(r)}{H_{0}^{2} r^{3}}}\right)+\frac{\mu}{4 \pi} \frac{4 \pi r^{3} \rho(r)-3 M(r)}{r^{3} \sqrt{1+\frac{8 G \epsilon^{2} M(r)}{H_{0}^{2} r^{3}}}}\right] .
$$


The physical meaning of $\mu$ and $\epsilon$ is understood as follows. In the limit of large $r,(2.8)$ and (2.6) reduce to

$$
\frac{\triangle}{a^{2}} \varphi \simeq-\frac{3 G \zeta M(r)}{r^{3}}
$$

and

$$
\frac{\triangle}{a^{2}}\left(\frac{\Psi-\Phi}{2}\right) \simeq 4 \pi G_{\mathrm{eff}} \delta \rho,
$$

respectively, where $G_{\text {eff }}=G(1+\mu)$ is the effective gravitational constant in the linearized limit. Thus $\mu$ is the amplitude of the modification of gravity in the outer region of a halo in the linearized regime. On the other hand, in the limit of small $r,(2.7)$ gives

$$
\left|\frac{d \varphi}{d r}\right| \simeq \frac{1}{4 \lambda^{2}} \sqrt{\frac{8 G \lambda^{2} \zeta M(r)}{r}} \ll \frac{G M(r)}{r^{2}} \sim\left|\frac{d \Psi}{d r}\right|,\left|\frac{d \Phi}{d r}\right| .
$$

This means that the effect of the scalar field is screened due to the nonlinear interaction of the scalar field in the limit of small $r$, which is the Vainshtein mechanism. Hence, the basic equations (2.3), (2.4) and (2.6) reduce to those of general relativity when the scalar field is neglected.

Thus, the modified gravity effect appears in the linear regime at large radii, while the modified gravity effect is screened due to the nonlinear effect at small radii. The transition radius between the two regime is the Vainshtein radius $r_{V}$, which we defined by (cf. $[33,54]$ )

$$
r_{V} \equiv\left[8 G \lambda^{2} \zeta M_{\mathrm{vir}}\right]^{1 / 3}=\left[\frac{8 G \epsilon^{2} M_{\mathrm{vir}}}{H_{0}^{2}}\right]^{1 / 3},
$$

where $M_{\text {vir }}$ is the virial mass of a cluster (see below). Therefore, $\epsilon$ is the parameter of the Vainshtein radius $r_{V}$. In summary, Newtonian gravity is recovered due to the Vainshtein mechanism for $r \ll r_{V}$, while the gravity is modified for $r \gg r_{V}$. The Vainshtein radius is rewritten as

$$
r_{V}=13.4 \epsilon^{2 / 3}\left(\frac{M_{\mathrm{vir}}}{10^{15} \mathrm{M}_{\odot}}\right)^{1 / 3} h^{-1} \mathrm{Mpc} .
$$

In the limit $\mu \rightarrow 0$ or $\epsilon \rightarrow \infty$, Newtonian gravity is reproduced on all scales. The original galileon model corresponds to $\mu=0.26$ and $\epsilon=0.53$ ( $\mu=0.19$ and $\epsilon=0.43)$ at the redshift $0.32(0.47)$, respectively, which is the mean redshift of clusters of the observational data used below.

Figures 1 and 2 demonstrate typical behavior of the surface mass density (left panel) and the logarithmic slope of the surface mass density (right panel) as a function of $r_{\perp}$. The curves are the theoretical model parametrized by $\mu$ and $\epsilon$, while the points with the error bars show the observational data by Umetsu et al. in $[75,76]$, which is obtained by averaging over four massive clusters. In figure 1, the theoretical curves assume the different values of $\mu$ with fixing $\epsilon$ and the other parameters for the halo density profile, as described in the panels, where we assumed the NFW density profile (see the next section). This figure demonstrates how the theoretical curve depends on $\mu$. As $r_{\perp}$ becomes large, the amplitude of $\Sigma_{S}\left(r_{\perp}\right)$ is enhanced for positive $\mu$, while it is suppressed for negative $\mu$. The (black) solid curve is 
Newtonian gravity, while the (magenta) dot and long-dashed curve is the original galileon model. Figure 2 is the same as figure 1 but with adopting the different theoretical values. Here the different values of $\epsilon$ are assumed while $\mu$ and the other parameters for the density profile are fixed, as described in this figure. As $\epsilon$ describes the Vainshtein radius $r_{V}$, within which Newtonian gravity is recovered, $\Sigma_{\mathrm{S}}\left(r_{\perp}\right)$ deviates from Newtonian gravity even at small radii for small $\epsilon$ (small $r_{V}$ ), while $\Sigma_{\mathrm{S}}\left(r_{\perp}\right)$ deviates from Newtonian gravity only at large radii for large $\epsilon$ (large $r_{V}$ ). For the case $\mu<0, \Sigma_{\mathrm{S}}\left(r_{\perp}\right)$ deviates from the line of $\mu=0$ in the opposite side of the case $\mu>0$.
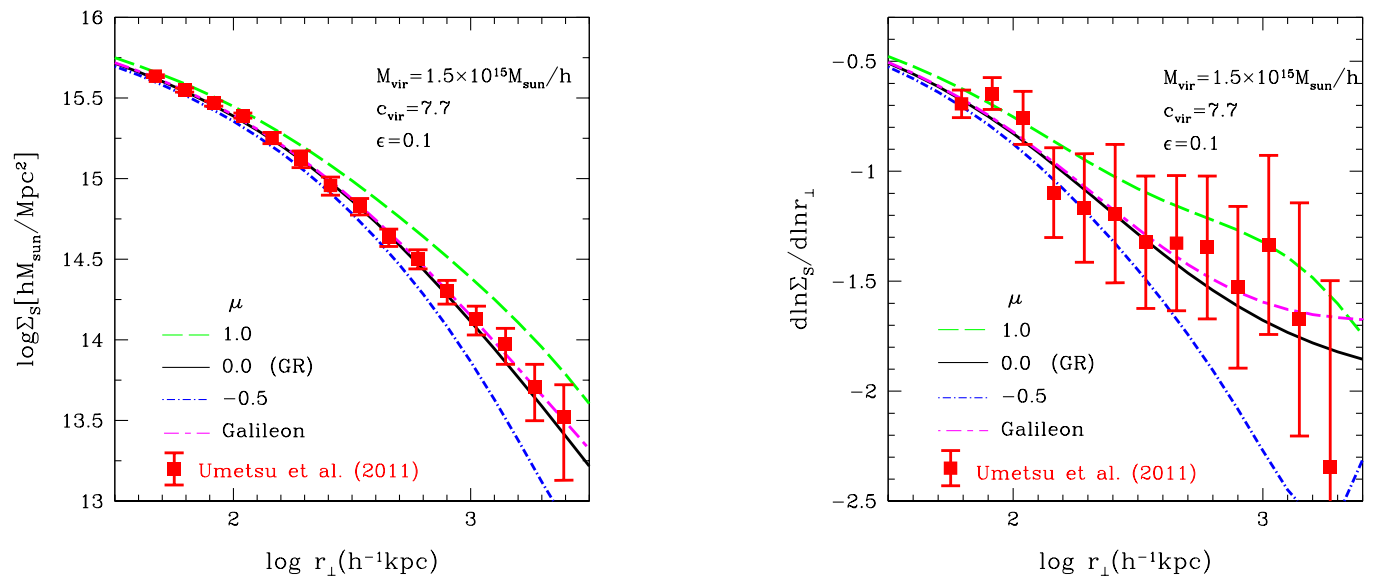

Figure 1. Surface mass density $\Sigma_{\mathrm{S}}\left(r_{\perp}\right)$ (left panel) and the logarithmic slope $d \ln \Sigma_{\mathrm{S}} / d \ln r_{\perp}$ (right panel) as function of $r_{\perp}$. The data with the error bar is from Umetsu et al. [75, 76], while the curves are the theoretical modeling. The (magenta) dot and long-dash curve is the original galileon model ( $\mu=0.26, \epsilon=0.53)$, but the other curves assume the same value of $\epsilon=0.1$ but the different values of $\mu=1$ (green dashed curve), 0 (black solid curve), and -0.5 (blue dot and short-dashed curve), respectively. Note that $\mu=0$ is Newtonian gravity. We here adopted the NFW profile with fixing $M_{\mathrm{vir}}=1.5 \times 10^{15} \mathrm{M}_{\odot} / h$ and $c_{\mathrm{vir}}=7.7$.

\section{Halo density profiles and differential surface mass density}

The halo density profile has been investigated as an important consequence of the cold dark matter paradigm, which is a key component of the universe. Lots of works have been done on the halo density profile with cosmological N-body simulations (e.g. [83-91]). The NFW universal profile $[59,60]$ is reported to work well, but the generalized NFW (gNFW) profile has also been studied [92-94]. Some studies indicate that the Einasto profile [95] better fits the inner cusps [96, 97, 99].

For predicting the surface mass density $\Sigma_{\mathrm{S}}\left(r_{\perp}\right)$, we need to determine the halo density profile $\rho(r)$. In the present paper, we adopt the halo density profiles described in this section, ${ }^{2}$ whose validity is suggested from N-body simulations. The halo density profiles could be affected by the modification of gravity, however, we adopt the same profile irrespective of gravity model, whose validity is partially suggested by N-body simulations for the DGP model and $f(R)$ model $[46,51]$. In this section, after briefly reviewing cold dark matter

\footnotetext{
${ }^{2}$ We only consider the contribution of the cluster halo profile itself (1-halo term) to $\Sigma_{\mathrm{S}}\left(r_{\perp}\right)$, and we neglect the neighboring halos (2-halo term), which is only important at large $r_{\perp}$ [94].
} 

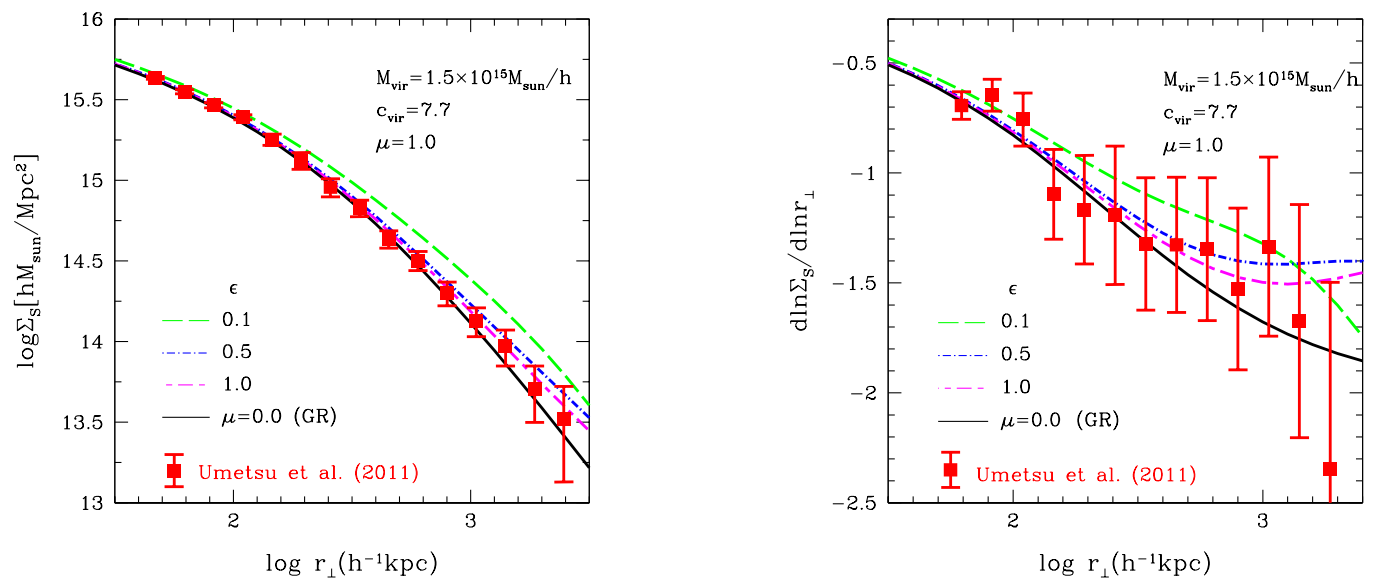

Figure 2. Same figure as figure 1 but with different theoretical models. The black solid curve is Newtonian gravity $\mu=0$, the other curves assume the same value of $\mu=1.0$ and the different values of $\epsilon=0.1$ (green dashed curve), 0.5 (blue dot short-dashed), and 1.0 (magenta dot long-dashed), respectively. Note that the curves approach Newtonian gravity as $\epsilon$ becomes large. We adopted the same NFW profile as figure $1, M_{\mathrm{vir}}=1.5 \times 10^{15} \mathrm{M}_{\odot} / h$ and $c_{\mathrm{vir}}=7.7$.
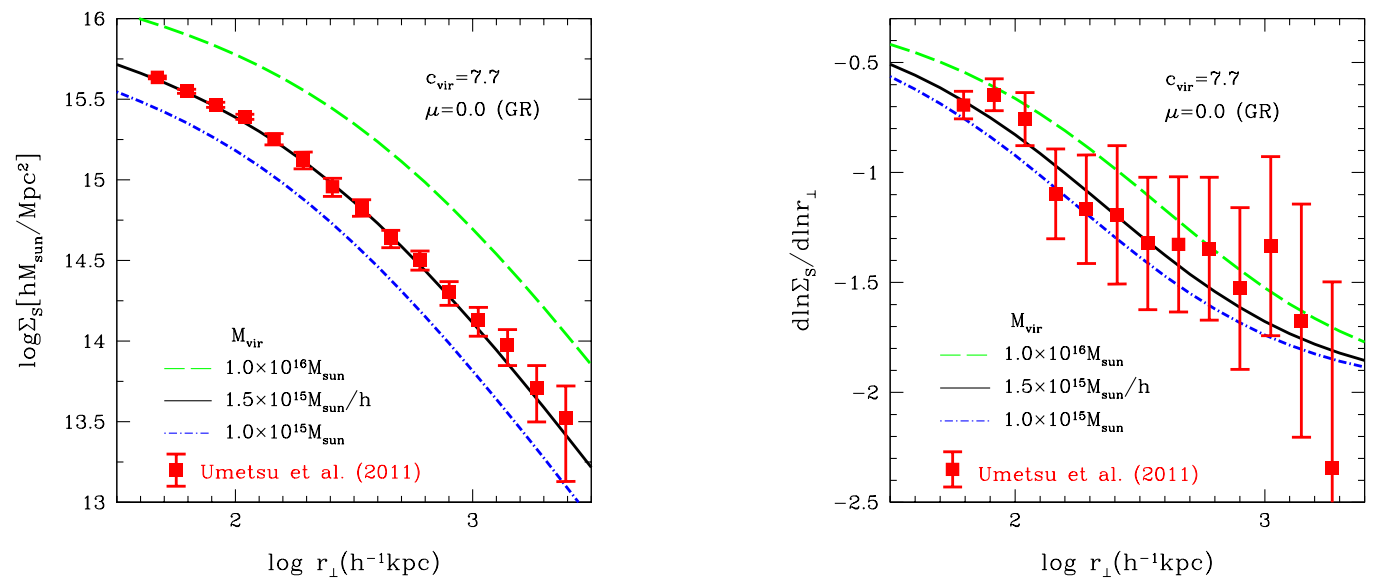

Figure 3. Surface mass density $\Sigma_{\mathrm{S}}\left(r_{\perp}\right)$ (left panel) and the logarithmic slope $d \ln \Sigma_{\mathrm{S}} / d \ln r_{\perp}$ (right panel) as function of $r_{\perp}$. The data with the error bar is from Umetsu et al. [75, 76], while the curves are the theoretical models assuming Newtonian gravity $\mu=0$ and the NFW profile with $c_{\mathrm{vir}}=7.7$ and $M_{\text {vir }}=1.0 \times 10^{16} \mathrm{M}_{\odot}$ (green dashed), $1.5 \times 10^{15} \mathrm{M}_{\odot} / h$ (black solid), and $1.0 \times 10^{15} \mathrm{M}_{\odot}($ blue dot-dashed), respectively.

(CDM) halo density profiles (see e.g., [97]), we demonstrate how the observed surface mass density depends on the modified gravity. This indicates the potential ability of testing the gravity theory with the halo density profile.

\subsection{NFW profile}

We first consider the NFW profile $[59,60]$

$$
\rho(r)=\frac{\rho_{s}}{\left(r / r_{s}\right)\left(1+r / r_{s}\right)^{2}},
$$



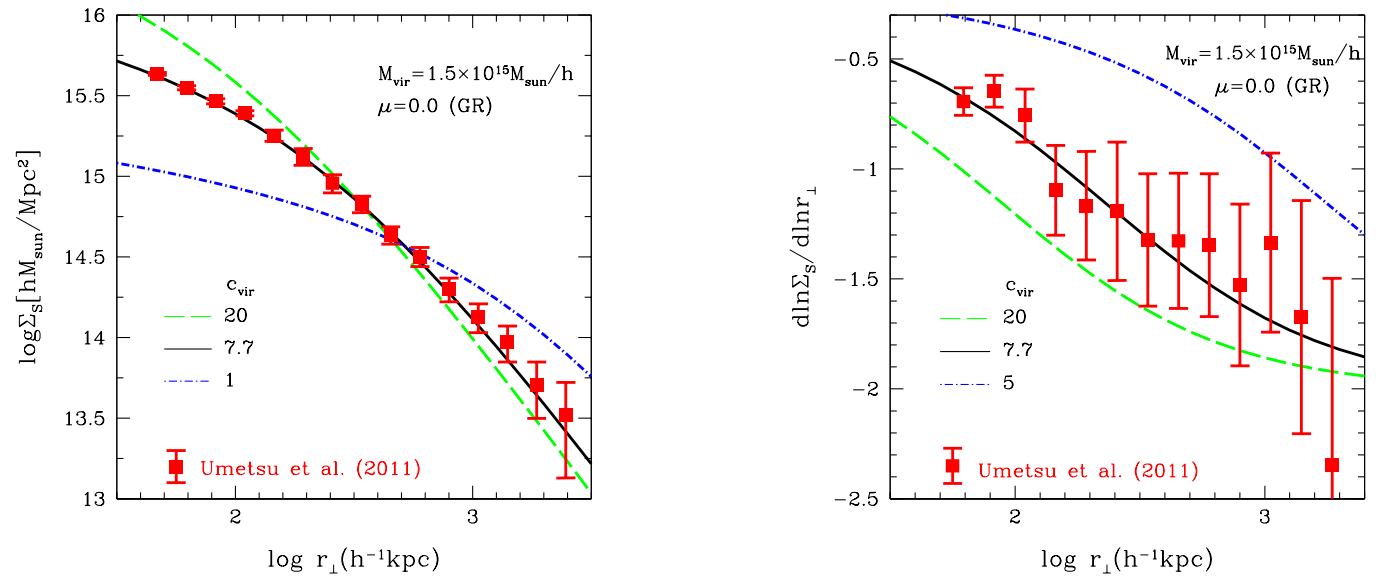

Figure 4. Same figure as figure 3 but with different values $c_{\mathrm{vir}}=20$ (green dashed curve), $c_{\mathrm{vir}}=7.7$ (black solid curve), and $c_{\mathrm{vir}}=1.0$ (blue dot-dashed curve), with $M_{\mathrm{vir}}=1.5 \times 10^{15} \mathrm{M}_{\odot} / h$ fixed. Here we assumed Newtonian gravity $\mu=0$ and the NFW profile.
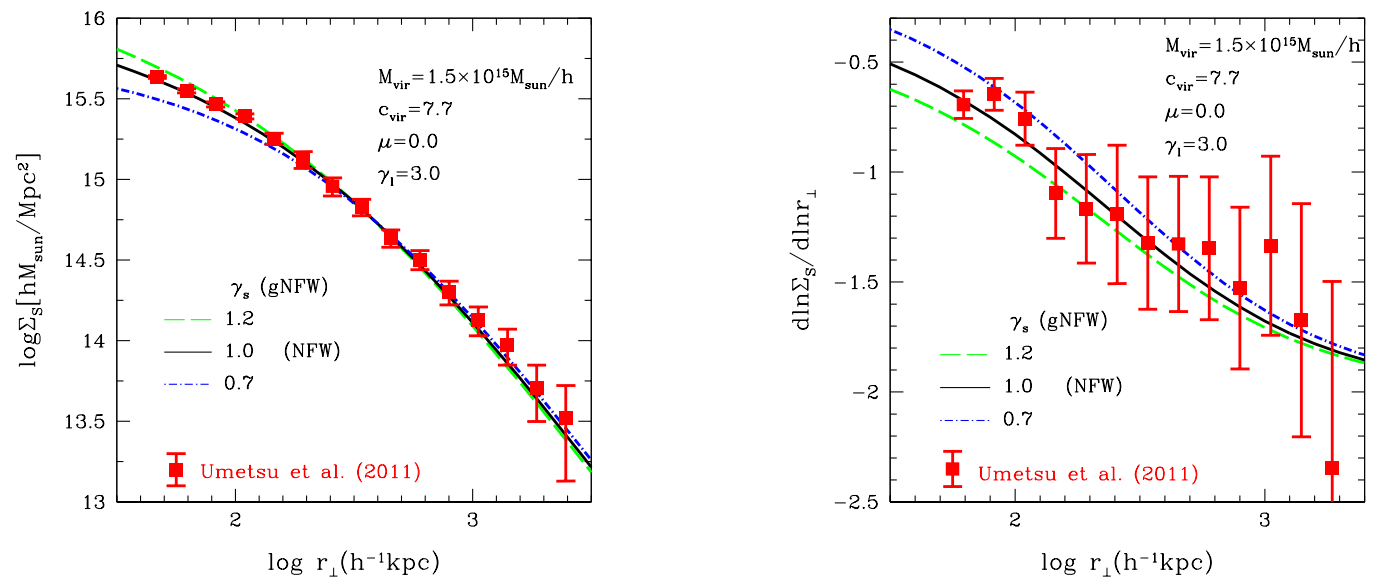

Figure 5. Same figure as figure 3 but for the gNFW profile with $\gamma_{s}=1.2$ (green dashed curve), $\gamma_{s}=1$ (black solid curve), and $\gamma_{s}=0.7$ (blue dot-dashed curve), respectively, with $\gamma_{l}=3$ fixed. Note that $\gamma_{s}=1$ is the NFW profile. The other parameters are fixed as $M_{\mathrm{vir}}=1.5 \times 10^{15} \mathrm{M}_{\odot} / h, c_{\mathrm{vir}}=7.7$, and $\mu=0$.

where $\rho_{s}=4 \rho\left(r_{s}\right)$ is the characteristic density, $r_{s}$ is the characteristic radius where the slope of the density profile changes. We define the virial cluster mass and the concentration parameter by

$$
\begin{aligned}
M_{\mathrm{vir}} & =\frac{4 \pi r_{\mathrm{vir}}^{3} \rho_{\mathrm{cr}}\left(z_{l}\right) \Delta_{\mathrm{vir}}}{3}, \\
c_{\mathrm{vir}} & =\frac{r_{\mathrm{vir}}}{r_{s}},
\end{aligned}
$$

respectively, which can be used as the parameters of the NFW profile instead of $\rho_{s}$ and $r_{s}$, where $r_{\text {vir }}$ is the virial radius, $\Delta_{\text {vir }}$ is the virial overdensity, and $\rho_{\mathrm{cr}}\left(z_{l}\right)=\rho_{\mathrm{cr}, 0} H^{2}\left(z_{l}\right) / H_{0}^{2}$ is the critical density. We take the value $\Delta_{\text {vir }}=120$. Note that the virial overdensity and the critical density depend on the cosmological background, which we do not specify explicitly. 

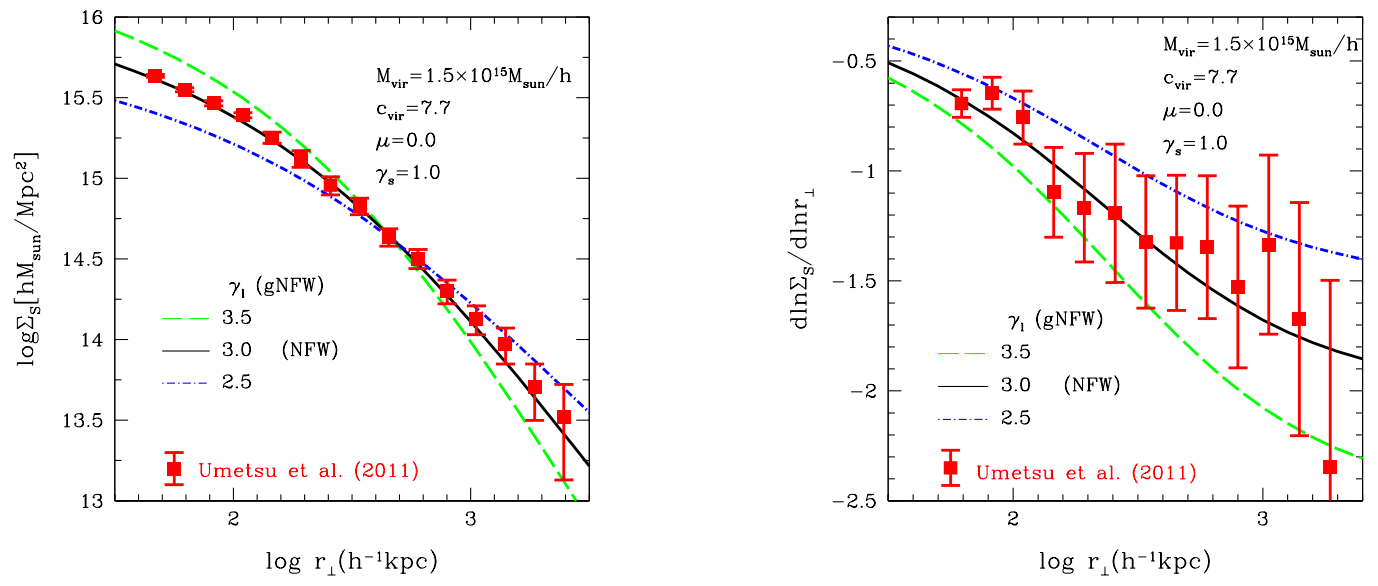

Figure 6. Same figure as figure 3 but for the gNFW profile with $\gamma_{l}=3.5$ (green dashed curve), $\gamma_{l}=3$ (black solid curve), and $\gamma_{l}=2.5$ (blue dot-dashed curve), respectively, with $\gamma_{s}=1$ fixed. Note that $\gamma_{l}=3$ is the NFW profile. The other parameters are fixed as $M_{\mathrm{vir}}=1.5 \times 10^{15} \mathrm{M}_{\odot} / h, c_{\mathrm{vir}}=7.7$, and $\mu=0$.
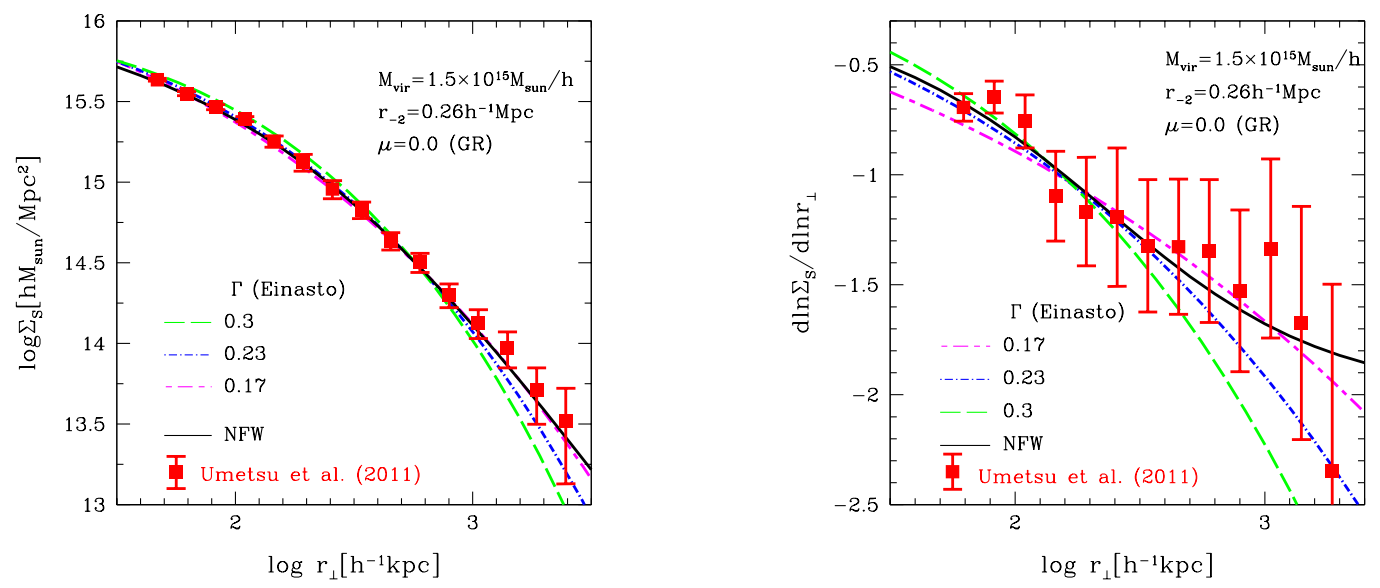

Figure 7. Same figure as figure 3 but for the Einasto profile with $\Gamma=0.3$ (green dashed curve), $\Gamma=0.23$ (blue dot short-dashed curve), and $\Gamma=0.17$ (magenta dot long-dashed curve), and with $r_{-2}=0.26 h^{-1} \mathrm{Mpc}$ fixed. The mass parameter is fixed $M_{\mathrm{vir}}=1.5 \times 10^{15} \mathrm{M}_{\odot} / h$, and we assumed Newtonian gravity $\mu=0$. For comparison, we also plot the NFW profile (black solid curve).

However, when $M_{\text {vir }}$ and $c_{\text {vir }}$ are marginalized over, the dependence on the cosmological background is not necessarily specified because it is absorbed by the redefinition of $M_{\text {vir }}$ and $c_{\text {vir }}$.

Figure 3 shows the surface mass density $\Sigma_{\mathrm{S}}\left(r_{\perp}\right)$ (left panel) and its logarithmic slope $d \ln \Sigma_{\mathrm{S}} / d \ln r_{\perp}$ (right panel), where we adopted the different values of $M_{\text {vir }}$ with the other parameters fixed, which are described therein. Here the concentration parameter is fixed $c_{\mathrm{vir}}=7.7$. Note that $\mu=0$ means Newtonian gravity. $M_{\mathrm{vir}}$ changes the amplitude of $\Sigma_{\mathrm{S}}\left(r_{\perp}\right)$. Figure 4 is the same figure as 3 , but with adopting the different values of $c_{\mathrm{vir}}$ and fixing the other parameter. Here we fixed the virial mass as $M_{\text {vir }}=1.5 \times 10^{15} \mathrm{M}_{\odot} / h$. The slope of the surface mass density significantly depends on $c_{\mathrm{vir}}$. 

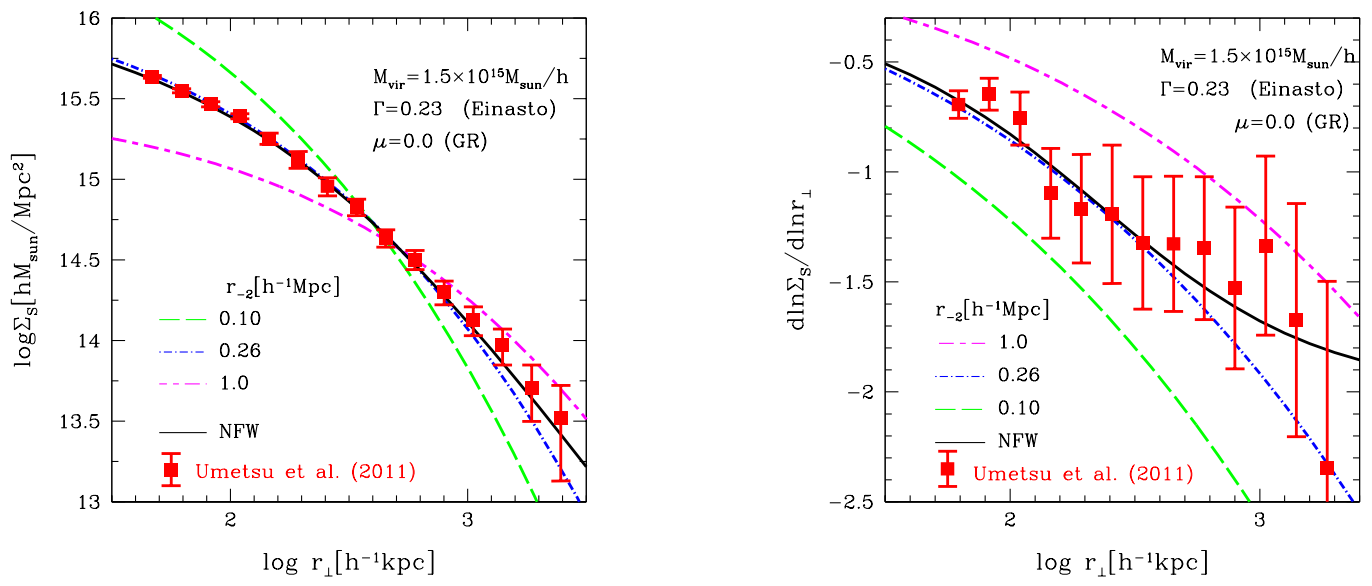

Figure 8. Same figure as figure 3 but for the Einasto profile wit $r_{-2}=0.1 h^{-1} \mathrm{Mpc}$ (green dashed curve), $0.22 h^{-1} \mathrm{Mpc}$ (blue dot short-dashed curve), and $1.0 h^{-1} \mathrm{Mpc}$ (magenta dot long-dashed curve), respectively, with $\Gamma=0.23$ fixed. The mass parameter is fixed $M_{\text {vir }}=1.5 \times 10^{15} \mathrm{M}_{\odot} / h$, and we assumed Newtonian gravity $\mu=0$. The black solid curve is the NFW profile.

As we will describe in the next section, we compare the theoretical model with observations by introducing the chi square statistics (see expression (4.1)). Within Newtonian gravity, the best-fit value of the chi-squared is 5.8 for 13 degrees of freedom (DOF), which we find for $M_{\text {vir }}=1.6 \times 10^{15} \mathrm{M}_{\odot} / h$ and $c_{\text {vir }}=7.9$. (see the top line of table 1 ).

\section{2 gNFW profile}

The generalized parametrization of the NFW model can be written in the form (e.g., [92-94])

$$
\rho(r)=\frac{\rho_{s}}{\left(r / r_{s}\right)^{\gamma_{s}}\left(1+r / r_{s}\right)^{\gamma_{l}-\gamma_{s}}} .
$$

The logarithmic slope of this density profile is defined

$$
\gamma_{3 D}(r) \equiv-\frac{d \ln \rho(r)}{d \ln r}
$$

which reduces to $\gamma_{s}$ for $r \ll r_{s}$, and to $\gamma_{l}$ for $r \gg r_{s}$. The NFW profile is reproduced for $\left(\gamma_{s}, \gamma_{l}\right)=(1,3)$. Cosmological N-body simulations indicate that an inner logarithmic slope of the density profile $\gamma_{3 D}(r) \lesssim 1.2$ and an asymptotic outer slope $\gamma_{3 D}(r) \gtrsim 2.5$ [97]. We introduce the radius $r_{-2}$ at which the outer slope is isothermal, i.e., $\gamma_{3 D}\left(r_{-2}\right)=2$. For the gNFW profile, $r_{-2}=r_{s}\left(2-\gamma_{s}\right) /\left(\gamma_{l}-2\right)$ and the corresponding concentration parameter is $c_{-2} \equiv r_{\text {vir }} / r_{-2}=c_{\text {vir }}\left(\gamma_{l}-2\right) /\left(2-\gamma_{s}\right)$.

Figure 5 shows $\Sigma_{\mathrm{S}}\left(r_{\perp}\right)$ and $d \ln \Sigma_{\mathrm{S}} / d \ln r_{\perp}$ adopting different values of $\gamma_{s}$ with the other parameters fixed as $\gamma_{l}=3.0, M_{\mathrm{vir}}=1.5 \times 10^{15} \mathrm{M}_{\odot} / h, c_{\mathrm{vir}}=7.7$, and $\mu=0$. The effect of changing $\gamma_{s}$ appears only inside halo. Figure 6 is the same as figure 5 , but with adopting the different values of $\gamma_{l}$ with the other parameters fixed as $\gamma_{s}=1.0, M_{\text {vir }}=1.5 \times 10^{15} \mathrm{M}_{\odot} / h$, $c_{\mathrm{vir}}=7.7$, and $\mu=0$. The behavior of $\Sigma_{\mathrm{S}}\left(r_{\perp}\right)$ is similar to that of figure 4 , which causes a degeneracy in the parameter space. We find the best-fit value of the chi-squared $\chi^{2} / \mathrm{DOF}=$ $5.5 / 13$ within Newtonian gravity for $\left(\gamma_{s}, \gamma_{l}\right)=(0.7,2.8)$, using the surface mass density data in [76]. This value of the chi-squared is slightly better than the best-fit value $\chi^{2} / \mathrm{DOF}=$ 5.8/13 for the NFW profile with $\left(\gamma_{s}, \gamma_{l}\right)=(1,3)$. The best-fit value for the gNFW profile with $\left(\gamma_{s}, \gamma_{l}\right)=(1.1,3.1)$ is $\chi^{2} / \mathrm{DOF}=6.2 / 13$ within Newtonian gravity (see table 1 ). 


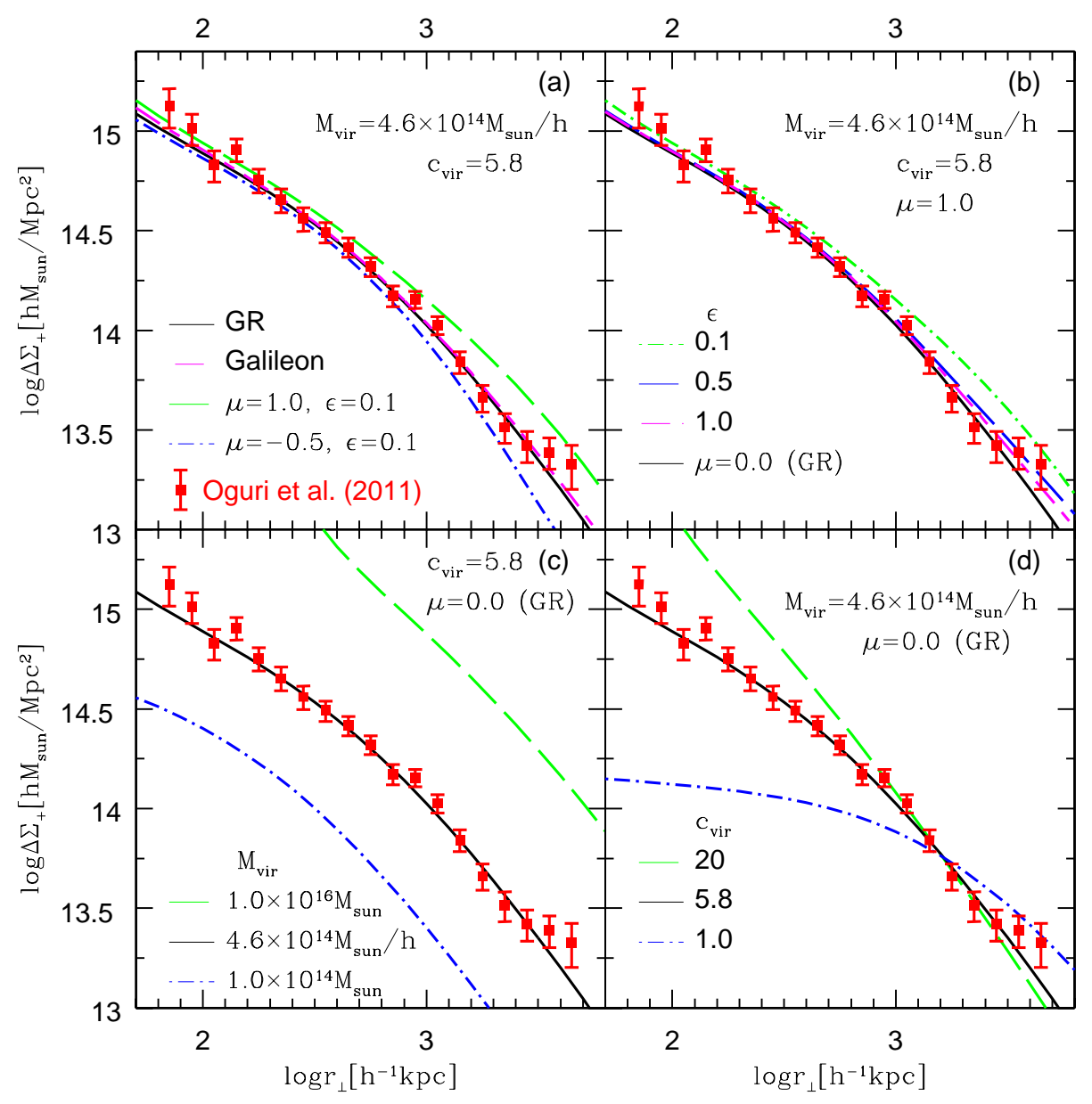

Figure 9. Differential surface mass density $\Delta \Sigma_{+}\left(r_{\perp}\right)$ in as a function of $r_{\perp}$. The data with the error bar is the result by Oguri et al. [77]. In the panel (a), the curves adopt the different values of $\mu=1.0$ (green dashed curve), $\mu=-0.5$ (blue dot short-dashed curve), and $\mu=0$ (black solid curve), respectively, with $\epsilon=0.1$ fixed. In this panel the NFW profile with $M_{\text {vir }}=4.6 \times 10^{14} \mathrm{M}_{\odot} / h$ and $c_{\mathrm{vir}}=5.8$ is adopted. The panel (b) is the same as the panel (a), but we adopted the different values of $\epsilon=0.1$ (green dashed curve), $\epsilon=0.5$ (blue dot short-dashed curve), and $\epsilon=1.0$ (magenta long-dashed curve), respectively, with $\mu=1.0$ fixed. The other parameters of $M_{\mathrm{vir}}$ and $c_{\mathrm{vir}}$ are the same as those of the panel (a). The panel (c) is the same as the panel (a), but we adopted the different values of $M_{\text {vir }}=1.0 \times 10^{16} \mathrm{M}_{\odot}$ (green dashed curve), $4.6 \times 10^{14} \mathrm{M}_{\odot} / h$ (black solid curve), and $1.0 \times 10^{14} \mathrm{M}_{\odot}$ (blue dot-dashed curve), respectively, for the NFW profile. The other parameters are fixed as $\mu=0$ and $c_{\text {vir }}=5.8$. The panel (d) is the same as the panel (c), but adopted the different values of $c_{\mathrm{vir}}=20$ (green dashed curve), 5.8 (black solid curve), and 1.0 (blue dot-dashed curve), respectively. The other parameters are fixed as $M_{\mathrm{vir}}=4.6 \times 10^{14} \mathrm{M}_{\odot}$ and $\mu=0.0$.

\subsection{Einasto profile}

Finally, we consider the Einasto profile [95]

$$
\rho(r)=\rho_{-2} \exp \left(-\frac{2}{\Gamma}\left[\left(\frac{r}{r_{-2}}\right)^{\Gamma}-1\right]\right),
$$




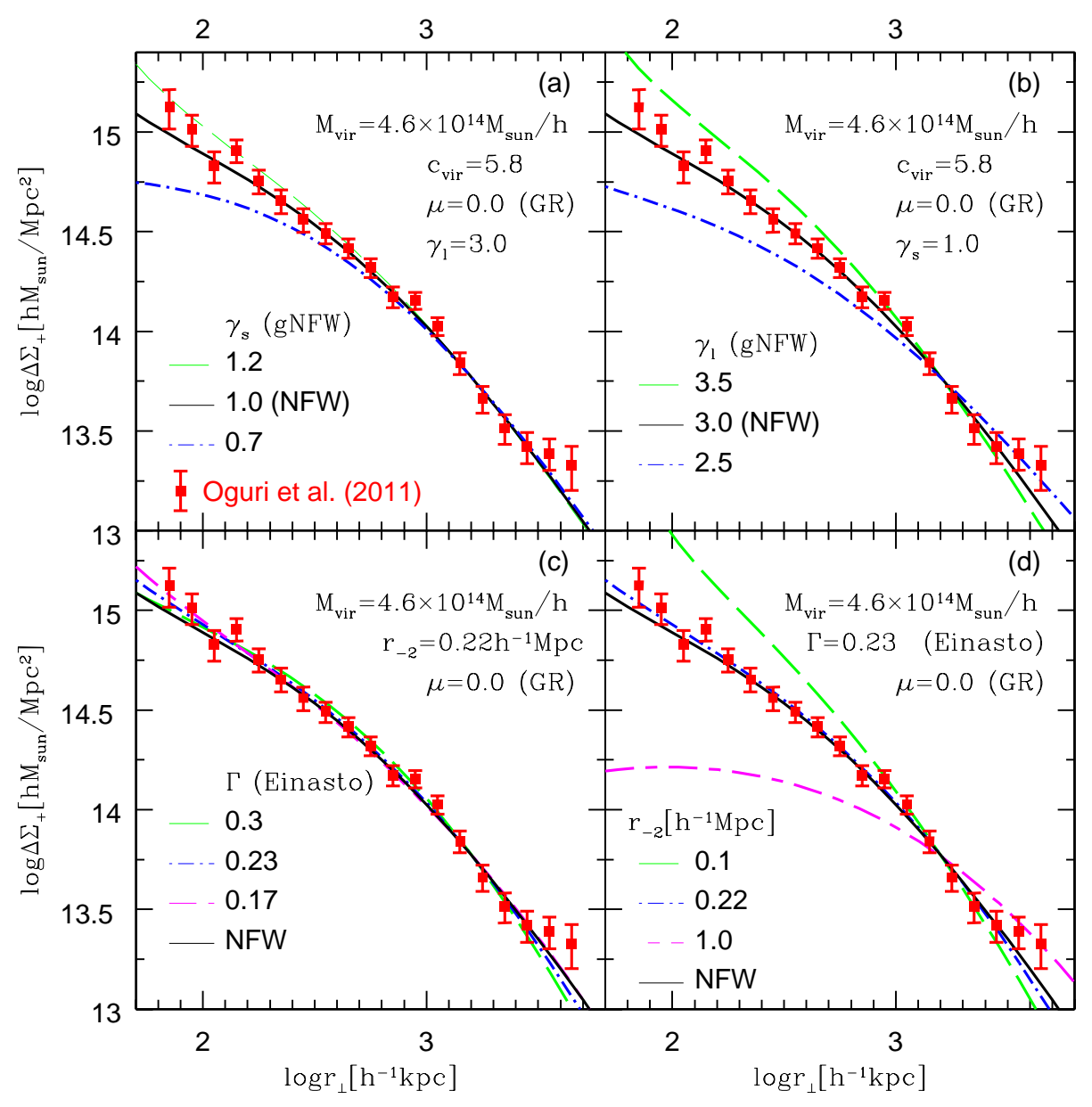

Figure 10. Same figure as figure 9 but with different theoretical models. The panels (a) and (b) assume the gNFW profile, and (c) and (d) do the Einasto profile, respectively. The panel (a) adopted the gNFW profile with $\gamma_{s}=1.2$ (green dashed curve), $\gamma_{s}=0.7$ (blue dot-dashed curve), and $\gamma_{s}=1$ (black solid curve), respectively. The other parameters are fixed as $\gamma_{l}=3, M_{\mathrm{vir}}=4.6 \times 10^{14} \mathrm{M}_{\odot} / h$, $c_{\mathrm{vir}}=5.8$, and $\mu=0$. In this panel $\gamma_{s}=1$ is equivalent to the NFW profile. The panel (b) is the same as the panel (a), but with $\gamma_{l}=3.5$ (green dashed curve), $\gamma_{l}=2.5$ (blue dot-dashed curve), and $\gamma_{l}=3$, respectively. In this panel, we fixed $\gamma_{s}=1$, then $\gamma_{l}=3$ is equivalent to the NFW profile. The panel (c) adopted the Einasto profile, with $\Gamma=0.3$ (green dashed curve), $\Gamma=0.23$ (blue dot short-dashed curve), and $\Gamma=0.17$ (magenta dot long-dashed curve), respectively. The other parameters are fixed $r_{-2}=0.22 h^{-1} \mathrm{Mpc}, M_{\mathrm{vir}}=4.6 \times 10^{14} \mathrm{M}_{\odot} / h$, and $\mu=0$. The panel (d) is the same as the panel (c), but with $r_{-2}=0.1 h^{-1} \mathrm{Mpc}$ (green dashed curve), $0.22 h^{-1} \mathrm{Mpc}$ (blue dot short-dashed curve), and $1.0 h^{-1} \mathrm{Mpc}$ (magenta dot long-dashed curve), respectively, while $\Gamma=0.23$ fixed.

where $r_{-2}$ and $\rho_{-2}$ are the radius and the density at which $\rho(r) \propto r^{-2}$, which means $\gamma_{3 D}\left(r_{-2}\right)=2$ and $\rho_{-2}=\rho\left(r_{-2}\right)$. The authors in [88] claimed that CDM halos can be more properly described by the Einasto profile than the NFW profile, using Millennium simulation. They also claimed that the best-fit value of $\Gamma$ increases gradually with the increase of the virial mass, from $\Gamma \sim 0.16$ for galaxy halos to $\Gamma \sim 0.3$ for the most massive clusters.

Figure 7 shows $\Sigma_{\mathrm{S}}\left(r_{\perp}\right)$ and $d \ln \Sigma_{\mathrm{S}} / d \ln r_{\perp}$ with adopting different values of $\Gamma$ but with 
the other parameters fixed $r_{-2}=0.26 h^{-1} \mathrm{Mpc}, M_{\mathrm{vir}}=1.5 \times 10^{15} \mathrm{M}_{\odot} / h$, and $\mu=0 . \Gamma$ changes the slope and the amplitude of $\Sigma_{S}\left(r_{\perp}\right)$ at large radii. Figure 8 is the same as figure 7 but with varying $r_{-2}$ with the other parameters fixed $\Gamma=0.23, M_{\text {vir }}=1.5 \times 10^{15} \mathrm{M}_{\odot} / h$, and $\mu=0$. The slope and the amplitude of $\Sigma_{\mathrm{S}}\left(r_{\perp}\right)$ strongly depend on $r_{-2}$.

We obtained the best-fit value of the chi-squared 9.9, 7.2, and 9.6 for $13 \mathrm{DOF}$ for the Einasto profile with $\Gamma=0.17,0.23$, and 0.3 , respectively (see table 1 ). Thus the Einasto profile does not fit the observational data better than the NFW profile or the gNFW profile within Newtonian gravity.

\subsection{Differential surface mass density}

Oguri et al. [77] obtained the differential surface mass density, which is defined by

$$
\Delta \Sigma_{+}\left(r_{\perp}\right) \equiv \Sigma_{\text {crit }} g_{+}\left(r_{\perp}\right)
$$

where $\Sigma_{\text {crit }}$ is defined by $(2.12), g_{+}\left(r_{\perp}\right)$ is the reduced shear

$$
g_{+}\left(r_{\perp}\right) \equiv \frac{\gamma_{+}\left(r_{\perp}\right)}{1-\kappa\left(r_{\perp}\right)}
$$

and the tangential shear is defined by

$$
\gamma_{+}\left(r_{\perp}\right)=\bar{\kappa}\left(<r_{\perp}\right)-\kappa\left(r_{\perp}\right)=\frac{\bar{\Sigma}_{\mathrm{S}}\left(<r_{\perp}\right)-\Sigma_{\mathrm{S}}\left(r_{\perp}\right)}{\Sigma_{\text {crit }}},
$$

with

$$
\bar{\Sigma}_{\mathrm{S}}\left(<r_{\perp}\right)=\frac{2}{r_{\perp}^{2}} \int_{0}^{r_{\perp}} d r_{\perp}^{\prime} r_{\perp}^{\prime} \Sigma_{\mathrm{S}}\left(r_{\perp}^{\prime}\right)
$$

In figures 9 and 10, we demonstrate the behavior of the differential surface mass density $\Delta \Sigma_{+}\left(r_{\perp}\right)$ as a function of $r_{\perp}$, comparing with the data in [77]. The panels (a), (b), (c) and (d) of figure 9 show how $\Delta \Sigma_{+}\left(r_{\perp}\right)$ depends on $\mu, \epsilon, M_{\text {vir }}$ and $c_{\text {vir }}$, respectively, adopting the NFW profile. The panels (a) and (b) of figure 10 show the dependence on $\gamma_{s}$ and $\gamma_{l}$ in the gNFW profile, while the panels (c) and (d) of figure 10 show the dependence on $\Gamma$ and $r_{-2}$ in the Einasto profile, respectively. The behaviors are very similar to those of the surface mass density described in the above subsections.

Similar to the surface mass density, we compare the theoretical prediction with the observational data by introducing the chi-squared, (4.8). We obtained the best-fit values of chi-squared for the NFW profile, the gNFW profile and the Einasto profile within Newtonian gravity as well as allowing the modified gravity, which are summarized in table 2 . We find the best-fit value of the chi-squared $\chi^{2} / \mathrm{DOF}=12.9 / 17$ for the NFW profile within Newtonian gravity, with the best-fit parameter $M_{\mathrm{vir}}=6.6 \times 10^{14} \mathrm{M}_{\odot} / h$ and $c_{\mathrm{vir}}=6.1$. This is consistent with the result by Oguri et al. [77]. The best-fit parameter is given $\bar{M}_{\text {vir }}=4.6 \times 10^{14} \mathrm{M}_{\odot} / h$ and $\bar{c}_{\mathrm{vir}}=5.7$, when we adopt the same definition for the virial mass $\bar{M}_{\mathrm{vir}}=4 \pi r_{\mathrm{vir}}^{3} \Delta(z) \rho_{m}(z)$.

The result is similar to that using the surface mass density in the point that the Einasto profile with large value of $\Gamma$ does not better fit the observational data than the NFW profile and the gNFW profile within Newtonian gravity. Thus the Einasto profile with $\Gamma=0.3$ is not favored within Newtonian gravity. 


\begin{tabular}{cc|rlr|rlr}
\hline \hline model & & Newtonian & gravity & Modified & gravity & \\
\hline & $\left(\gamma_{s}, \gamma_{l}\right)$ & $M_{\mathrm{vir}}\left[\mathrm{M}_{\odot}\right]$ & $c_{\mathrm{vir}}\left(c_{-2}\right)$ & $\chi_{\mathrm{GR}}^{2}$ & $M_{\mathrm{vir}}\left[\mathrm{M}_{\odot}\right]$ & $c_{\mathrm{vir}}\left(c_{-2}\right)$ & $\chi_{\mathrm{MG}}^{2}$ \\
\hline NFW & $(1,3)$ & $2.2 \times 10^{15}$ & 7.9 & 5.8 & $2.2 \times 10^{15}$ & 8.0 & 5.7 \\
gNFW & $(0.7,2.8)$ & $2.2 \times 10^{15}$ & $13.9(8.6)$ & 5.5 & $2.2 \times 10^{15}$ & $13.9(8.6)$ & 5.5 \\
gNFW & $(1.1,3.1)$ & $2.2 \times 10^{15}$ & $6.2(7.6)$ & 6.2 & $2.2 \times 10^{15}$ & $6.3(7.7)$ & 6.0 \\
\hline & $\Gamma$ & $M_{\mathrm{vir}}\left[\mathrm{M}_{\odot}\right]$ & $r_{-2}$ & $\chi_{\mathrm{GR}}^{2}$ & $M_{\mathrm{vir}}\left[\mathrm{M}_{\odot}\right]$ & $r_{-2}$ & $\chi_{\mathrm{MG}}^{2}$ \\
\hline Einasto & 0.17 & $2.5 \times 10^{15}$ & 0.30 & 9.9 & $2.9 \times 10^{15}$ & 0.35 & 8.7 \\
Einasto & 0.23 & $2.2 \times 10^{15}$ & 0.28 & 7.2 & $2.1 \times 10^{15}$ & 0.26 & 6.5 \\
Einasto & 0.3 & $1.9 \times 10^{15}$ & 0.26 & 9.6 & $1.6 \times 10^{15}$ & 0.22 & 5.6 \\
\hline \hline
\end{tabular}

Table 1. Best-fit value of the chi-squared of the surface mass density $\Sigma_{\mathrm{S}}\left(r_{\perp}\right)$ for the NFW profile, the gNFW profile and the Einasto profile. Here we fixed the values of $\gamma_{s}$ and $\gamma_{l}$ for the gNFW profile, and $\Gamma$ for the Einasto profile, as described in the table. $\chi_{\mathrm{GR}}^{2}$ is the best-fit value for each halo model when Newtonian gravity is assumed, and $M_{\mathrm{vir}}$ and $c_{\mathrm{vir}}$ or $r_{-2}$ (in unit of $h^{-1} \mathrm{Mpc}$ ) yield the best-fit value. $\chi_{\mathrm{MG}}^{2}$ is the best-fit value allowing the modified gravity model. The number of degrees of freedom (DOF) is 13 and 11 for $\chi_{\mathrm{GR}}^{2}$ and $\chi_{\mathrm{MG}}^{2}$, respectively.

\begin{tabular}{|c|c|c|c|c|c|c|c|}
\hline model & & Newtonian & gravity & & Modified & gravity & \\
\hline & $\left(\gamma_{s}, \gamma_{l}\right)$ & $M_{\mathrm{vir}}\left[\mathrm{M}_{\odot}\right]$ & $c_{\mathrm{vir}}\left(c_{-2}\right)$ & $\chi_{\mathrm{GR}}^{2}$ & $M_{\text {vir }}\left[\mathrm{M}_{\odot}\right]$ & $c_{\mathrm{vir}}\left(c_{-2}\right)$ & $\chi_{\mathrm{MG}}^{2}$ \\
\hline NFW & $(1,3)$ & $6.8 \times 10^{14}$ & 6.1 & 12.9 & $5.9 \times 10^{15}$ & 6.6 & 11.7 \\
\hline gNFW & $(0.7,2.8)$ & $6.8 \times 10^{14}$ & $10.9(6.7)$ & 13.1 & $6.1 \times 10^{15}$ & $11.7(7.2)$ & 12.2 \\
\hline gNFW & $(1.1,3.1)$ & $6.8 \times 10^{14}$ & $4.8(5.9)$ & 12.8 & $5.9 \times 10^{15}$ & $5.3(6.5)$ & 11.5 \\
\hline & $\Gamma$ & $M_{\text {vir }}\left[\mathrm{M}_{\odot}\right]$ & $r_{-2}$ & $\chi_{\mathrm{GR}}^{2}$ & $M_{\mathrm{vir}}\left[\mathrm{M}_{\odot}\right]$ & $r_{-2}$ & $\chi_{\mathrm{MG}}^{2}$ \\
\hline Einasto & 0.17 & $7.0 \times 10^{14}$ & 0.23 & 12.4 & $6.6 \times 10^{15}$ & 0.21 & 12.2 \\
\hline Einasto & 0.23 & $6.8 \times 10^{14}$ & 0.23 & 14.0 & $5.4 \times 10^{15}$ & 0.18 & 11.4 \\
\hline Einasto & 0.3 & $6.7 \times 10^{14}$ & 0.23 & 19.7 & $4.7 \times 10^{15}$ & 0.17 & 11.8 \\
\hline
\end{tabular}

Table 2. Same table as table 1 but for the differential surface mass density $\Delta \Sigma_{+}\left(r_{\perp}\right)$. The number of DOF is 17 and 15 for $\chi_{\mathrm{GR}}^{2}$ and $\chi_{\mathrm{MG}}^{2}$, respectively.

\section{Results - Comparison with observations -}

In this section we demonstrate the constraint on the modified gravity parameters $\mu$ and $\epsilon$ using the observational data. We first use the surface mass density $\Sigma_{\mathrm{S}}\left(r_{\perp}\right)$ and its logarithmic slope $d \ln \Sigma_{\mathrm{S}} / d \ln r_{\perp}$, obtained by Umetsu et al. through the accurate strong- and weaklensing measurements [76]. We define the chi-squared for the surface mass density by

$$
\left.\left.\chi^{2}\left(\mu, \epsilon, M_{\mathrm{vir}}, c_{\mathrm{vir}}\right) \equiv \sum_{i, j}\left[\Sigma_{\mathrm{S}}^{\text {theo }}\left(r_{\perp i}\right)-\Sigma_{\mathrm{S}}^{\mathrm{obs}}\left(r_{\perp i}\right)\right)\right] \operatorname{Cov}_{i j}^{-1}\left[\Sigma_{\mathrm{S}}^{\text {theo }}\left(r_{\perp j}\right)-\Sigma_{\mathrm{S}}^{\mathrm{obs}}\left(r_{\perp j}\right)\right)\right]
$$

where $\Sigma_{\mathrm{S}}^{\mathrm{obs}}\left(r_{\perp i}\right)$ and $\mathrm{Cov}_{i j}^{-1}$ are the observational surface mass density and the inverse matrix of the $1 \sigma$ covariance matrix, respectively, at the $i$-th projected radius $r_{\perp i}$, which are obtained by stacking individual full surface mass density of four high-mass clusters (A1689, A1703, 
$\mathrm{A} 370$, and $\mathrm{Cl0024+17)}$ at an average redshift $\left\langle z_{l}\right\rangle=0.32[75,76]$, and $\Sigma_{\mathrm{S}}^{\text {theo }}\left(r_{\perp i}\right)$ is the theoretically predicted surface mass density.

For comparison, we also compute the chi-squared for the logarithmic slope $d \ln \Sigma_{\mathrm{S}} / d \ln r_{\perp}$, which can be defined in the similar way to (4.1),

$$
\chi^{2}\left(\mu, \epsilon, c_{\mathrm{vir}}, M_{\mathrm{vir}}\right) \equiv \sum_{i} \frac{\left[d \ln \Sigma_{\mathrm{S}}^{\mathrm{theo}}\left(r_{\perp i}\right) / d \ln r_{\perp}-d \ln \Sigma_{\mathrm{S}}^{\mathrm{obs}}\left(r_{\perp i}\right) / d \ln r_{\perp}\right]^{2}}{\left[\Delta d \ln \Sigma_{\mathrm{S}}\left(r_{\perp i}\right) / d \ln r_{\perp}\right]^{2}},
$$

where $d \ln \Sigma_{\mathrm{S}}^{\text {theo }}\left(r_{\perp i}\right) / d \ln r_{\perp}$ and $d \ln \Sigma_{\mathrm{S}}^{\mathrm{obs}}\left(r_{\perp i}\right) / d \ln r_{\perp}$ are the theoretical value and the observational value, respectively, and $\Delta d \ln \Sigma_{\mathrm{S}}\left(r_{\perp i}\right) / d \ln r_{\perp}$ is the 1-sigma error of the data (see the right panel of figure 1).

The left panel of figure 11 shows the contour of $\Delta \chi^{2}$, to show the relative confidence level of $\mu$ and $\epsilon$ with respect to their best-fit values

$$
\Delta \chi^{2}(\mu, \epsilon) \equiv \chi^{2}\left(\mu, \epsilon, M_{\mathrm{vir}, \text { local min }}, c_{\mathrm{vir}, \text { local min }}\right)-\chi^{2}\left(\mu_{\min }, \epsilon_{\min }, M_{\mathrm{vir}, \min }, c_{\mathrm{vir}, \min }\right) .
$$

In (4.3), $\mu_{\min }, \epsilon_{\min }, M_{\mathrm{vir}, \min }$, and $c_{\mathrm{vir}, \min }$ denote their best-fit values which globally minimize the value of $\chi^{2}$, while $M_{\mathrm{vir}, \text { local min }}$ and $c_{\mathrm{vir}, \text { local min }}$ are the values that locally minimize the $\chi^{2}$ for a given set of values of $\mu$ and $\epsilon$, where we consider the range

$$
\begin{aligned}
10^{13} \mathrm{M}_{\odot} & \leq M_{\text {vir }} \leq 10^{16} \mathrm{M}_{\odot} \\
0.01 & \leq c_{\text {vir }} \leq 40
\end{aligned}
$$

and

$$
\begin{aligned}
0.1 & \leq \epsilon \leq 10, \\
-5.0 & \leq \mu \leq 5.0 .
\end{aligned}
$$

Our results does not depend on this choice significantly. In table 1 we listed the best-fit value of the chi-squared of (4.1) $\chi_{\mathrm{MG}}^{2}=\chi^{2}\left(\mu_{\mathrm{min}}, \epsilon_{\mathrm{min}}, M_{\mathrm{vir}, \min }, c_{\mathrm{vir}, \mathrm{min}}\right)$, for each halo model.

In the left panel of figure 11, the region sandwiched between the solid curves is the 1sigma allowed region with the chi-squared of the surface mass density (4.1), while the region between the dashed curves is the same but with the chi-squared of the logarithmic slope (4.2). Here we assumed the NFW profile. The small cross in this figure marks the value of the original galileon model, $\mu=0.26$ and $\epsilon=0.53$ at the redshift 0.32 in the left panel.

Note that we adopted the NFW profile in figure 11, while the panels in figure 12 assumed the different profiles. The panels (a) and (b) adopted the gNFW profile with $\left(\gamma_{s}, \gamma_{l}\right)=$ $(0.7,2.8)$ and $(1.1,3.1)$, respectively, while $(\mathrm{c})$ and $(\mathrm{d})$ adopted the Einasto profile with $\Gamma=$ 0.17 and 0.23 , respectively.

We also investigate the constraint from the data of the differential surface mass density $\Delta \Sigma_{+}\left(r_{\perp}\right)$, obtained by Oguri et al. [77]. We define the chi-squared for $\Delta \Sigma_{+}\left(r_{\perp}\right)$ by

$$
\chi^{2} \equiv \sum_{i} \frac{\left.\left[\Delta \Sigma_{+}^{\text {theo }}\left(r_{\perp i}\right)-\Delta \Sigma_{+}^{\mathrm{obs}}\left(r_{\perp i}\right)\right)\right]^{2}}{\sigma_{\mathrm{obs}}^{2}\left(r_{\perp i}\right)},
$$

where $\Delta \Sigma_{+}^{\text {obs }}\left(r_{\perp i}\right)$ and $\sigma_{\text {obs }}\left(r_{\perp i}\right)$ are the observed differential surface mass density and the $1 \sigma$ error for the $i$-th projected radius $r_{\perp i}$ in [77], and $\Delta \Sigma_{+}^{\text {theo }}\left(r_{\perp i}\right)$ is the theoretical differential surface mass density. The right panel of figure 11 is the contour of $\Delta \chi^{2}$ for the differential 


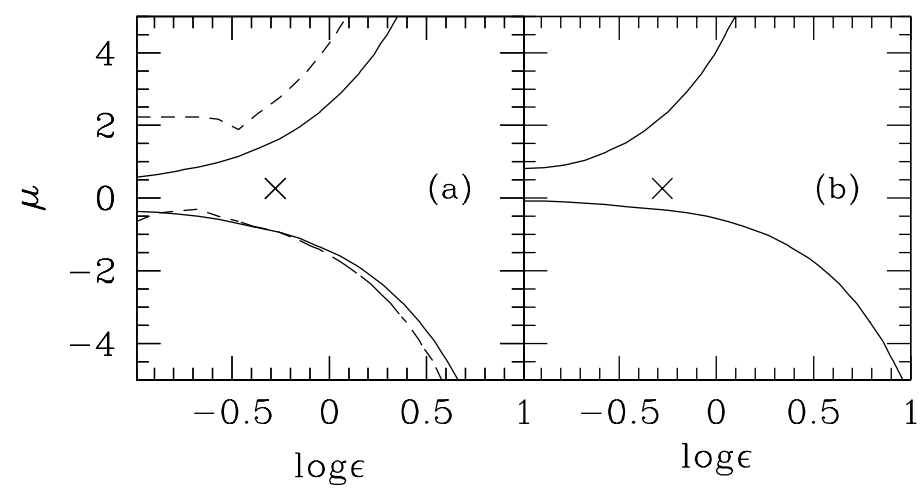

Figure 11. (a) 1-sigma confidence contour $\Delta \chi^{2}=2.3$ on the $\mu-\epsilon$ plane with the surface mass density $\Sigma_{\mathrm{S}}\left(r_{\perp}\right)$ (solid curve), and with the logarithmic slope of the surface mass density $d \ln \Sigma_{\mathrm{S} /} / d \ln r_{\perp}$ (dashed curve). The region sandwiched between a pair of two curves is statistically allowed at 1sigma level. The point marked by the cross corresponds to the original galileon model (see appendix B). (b) 1-sigma confidence contour $\Delta \chi^{2}=2.3$ on $\mu-\epsilon$ plane with the differential surface mass density $\Delta \Sigma_{+}\left(r_{\perp}\right)$. In these panels, the NFW profile is assumed.

surface mass density, defined in the same way as (4.3). The region sandwiched between the solid curves is the 1-sigma allowed region, and the point marked by the cross is the original galileon model, $\mu=0.19$ and $\epsilon=0.43$ at the redshift $z=0.47$. In the right panel of figure 11 , we adopted the NFW profile, and the best-fit value of the chi squared is noted in table 2 as $\chi_{\mathrm{MG}}^{2}$.

The panels in figure 13 are the same as the right panel of figure 11, but with adopting the different profiles. The panels (a) and (b) of figure 13 assume the gNFW profile with $\left(\gamma_{s}\right.$, $\left.\gamma_{l}\right)=(0.7,2.8)$ and $(1.1,3.1)$, while (c) and (d) adopt the Einasto profile with $\Gamma=0.17$ and 0.23 , respectively. Note that the panel (d) indicates that the modified gravity is favored than Newtonian gravity when the Einasto profile with large $\Gamma$ is assumed.

\section{Discussion}

Our main result is symbolically expressed by figure 11. From figure 11, Newtonian gravity, i.e., $\mu=0$ or $\epsilon \gg 1$, is favored, and the original galileon model is in the 1-sigma allowed region, which is not rejected. One can see that the data put a constraint $|\mu| \lesssim 1$ when $\epsilon \lesssim 1$, but the constraint on $\mu$ becomes weaker as $\epsilon$ becomes larger. For instance, $-0.4 \lesssim \mu \lesssim 0.6$ for $\epsilon=0.1$ and $-1.5 \lesssim \mu \lesssim 2.5$ for $\epsilon=1.0$.

The Vainshtein radius $r_{V}$ depends on $\epsilon$ and $M_{\text {vir }}$, from eq. (2.19). The method in the present paper cannot constrain the model in which the Vainshtein radius $r_{V}$ is larger than the cluster scale because the effect of the modified gravity becomes very weak inside the Vainshtein radius. The data we used is limited by $r \lesssim 3 h^{-1} \mathrm{Mpc}$ for the surface mass density, and by $r \lesssim 5 h^{-1} \mathrm{Mpc}$ for the differential surface mass density. These facts set a limit of constraining $\mu$ and $\epsilon$. On the other hand, when the Vainshtein radius $r_{V}$ is smaller than the cluster scales $r_{\text {vir }}$, the effect of the modified gravity appears inside the cluster scale. Therefore, we can put a rather tight constraint on $\mu$ for small $\epsilon$.

In tables 1 and 2 , respectively, the best-fit value of the chi-squared is listed for each halo model, using the surface mass density and the differential surface mass density. $\chi_{\mathrm{MG}}^{2}$ is the best-fit value allowing the modified gravity model. Note that $\chi_{\mathrm{MG}}^{2}$ is improved, compared 


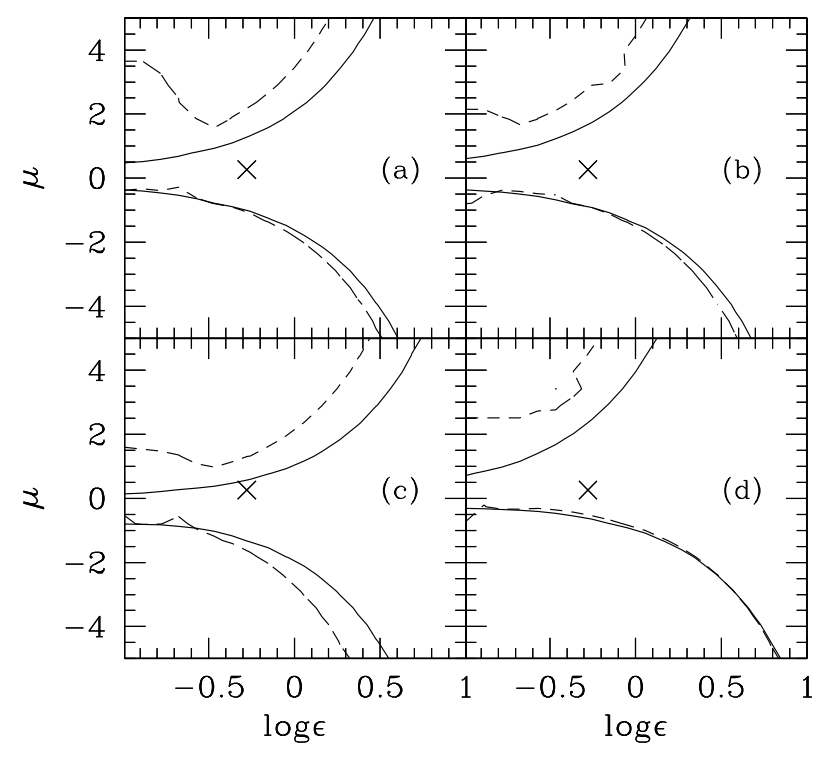

Figure 12. Each panel is the same as the left panel of figure 11, but adopted the different halo profile. The panels (a) and (b) adopted the gNFW profile with $\left(\gamma_{s}, \gamma_{l}\right)=(0.7,2.8)$ and $\left(\gamma_{s}, \gamma_{l}\right)=(1.1,3.1)$, while (c) and (d) adopted the Einasto profile with $\Gamma=0.17$ and $\Gamma=0.23$, respectively.

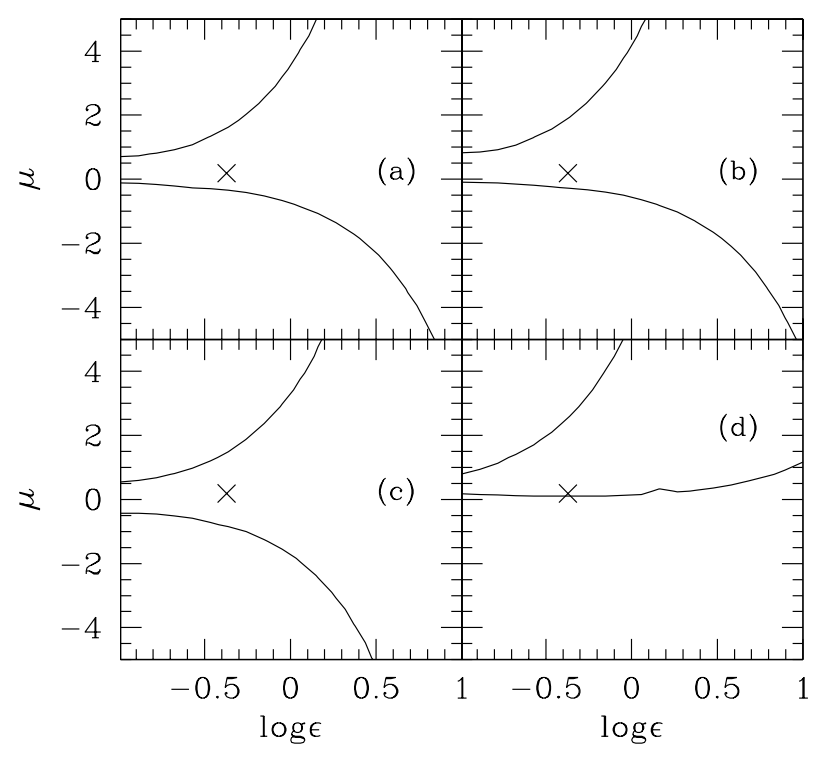

Figure 13. Same figure as figure 12 but with the differential surface mass density $\Delta \Sigma_{+}\left(r_{\perp}\right)$. 
with $\chi_{\mathrm{GR}}^{2}$, which is the best-fit value within Newtonian gravity. We see slight improvement for the NFW profile and the gNFW profile. This reflects that Newtonian gravity is favored from the data as long as the halo profile is the NFW profile or the gNFW profile.

Another point is that the best-fit value is significantly improved by allowing the modified gravity for the Einasto profile with $\Gamma=0.23$ and $\Gamma=0.3$. This can be explained as follows by using figures 14 and 15, which show how the fitting is improved by the modified gravity model. Figure 14 shows that the fitting of $\Sigma_{S}\left(r_{\perp}\right)$ is improved at large radii by the modified gravity model. Figure 15 shows that the fitting of $\Delta \Sigma_{+}\left(r_{\perp}\right)$ is improved at large radii as well as at smaller radii. Thus the modified gravity model is favored if the halo follows the Einasto profile with large value of $\Gamma$. This is the reason why the modified gravity is favored in the panel (d) of figure 13.

The above results rely mostly on the behavior of the theoretical curves in the outer region. Let us check the validity of our theoretical modeling in the outer region. The behavior in the outer region is determined by the halo density profile which we choose and the modified gravity effect of the scalar field. When the density profile is fixed, the modified gravity's effect of the scalar field is properly taken into account by using the expression (2.13). In the present paper, however, we assumed the halo density profiles irrespective of the modified gravity model. This point will need to be tested carefully, though there exists a supporting evidence in reference $[46,51]$. In the modeling of the halo density profile, we only considered the contribution of the cluster halo profile itself (1-halo term), and we neglect the neighboring halos (2-halo term), which will be only important at large radius [94].
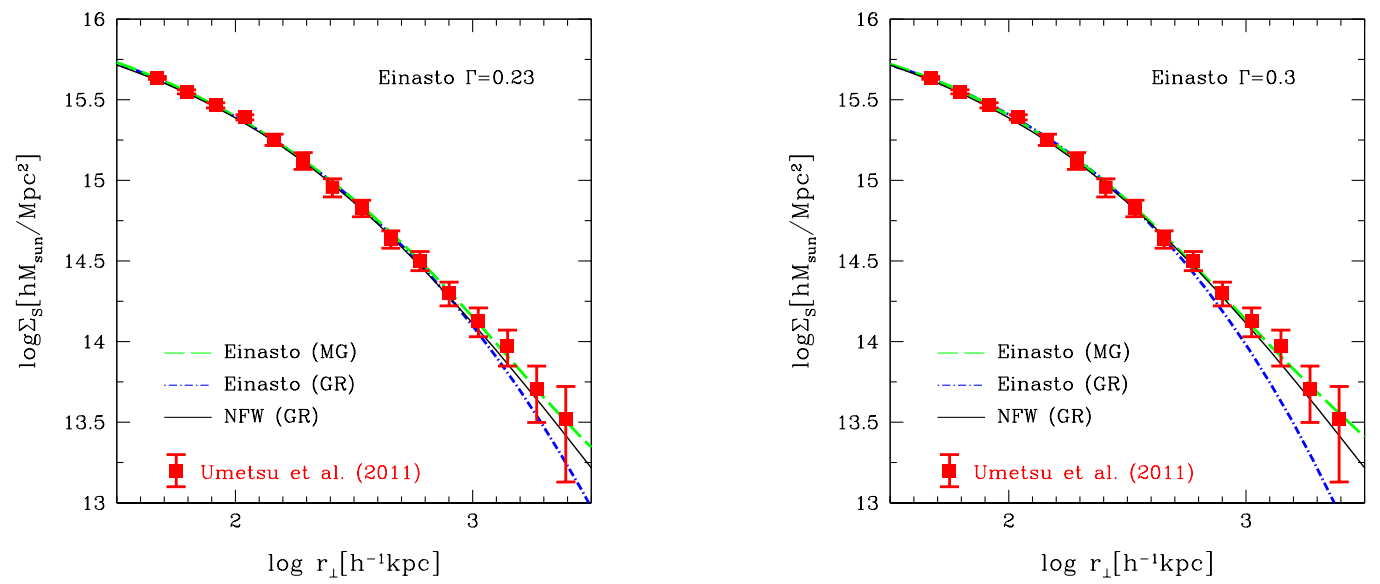

Figure 14. This figure compares the observed surface mass density $\Sigma_{\mathrm{S}}\left(r_{\perp}\right)$ and the theoretical curve assuming the Einasto profile with $\Gamma=0.23$ (left panel) and $\Gamma=0.3$ (right panel), respectively. In each panel, the blue dot and short-dashed curve is the best-fit curve within the Newtonian gravity, while the green dashed curve is the best-fit curve of the modified gravity. The solid curve assumes the NFW profile within the Newtonian gravity. The left (right) panel adopted $\Gamma=0.23(0.3)$, and the best-fit parameters for the modified gravity model are $\mu=5.0$ and $\epsilon=4.5(\mu=3.4$ and $\epsilon=0.69)$, respectively.

\section{Summary and Conclusion}

We investigated a constraint on the subclass of the generalized galileon model endowed with the Vainshtein mechanism, which includes a wide class of scalar-tensor gravity theories. 

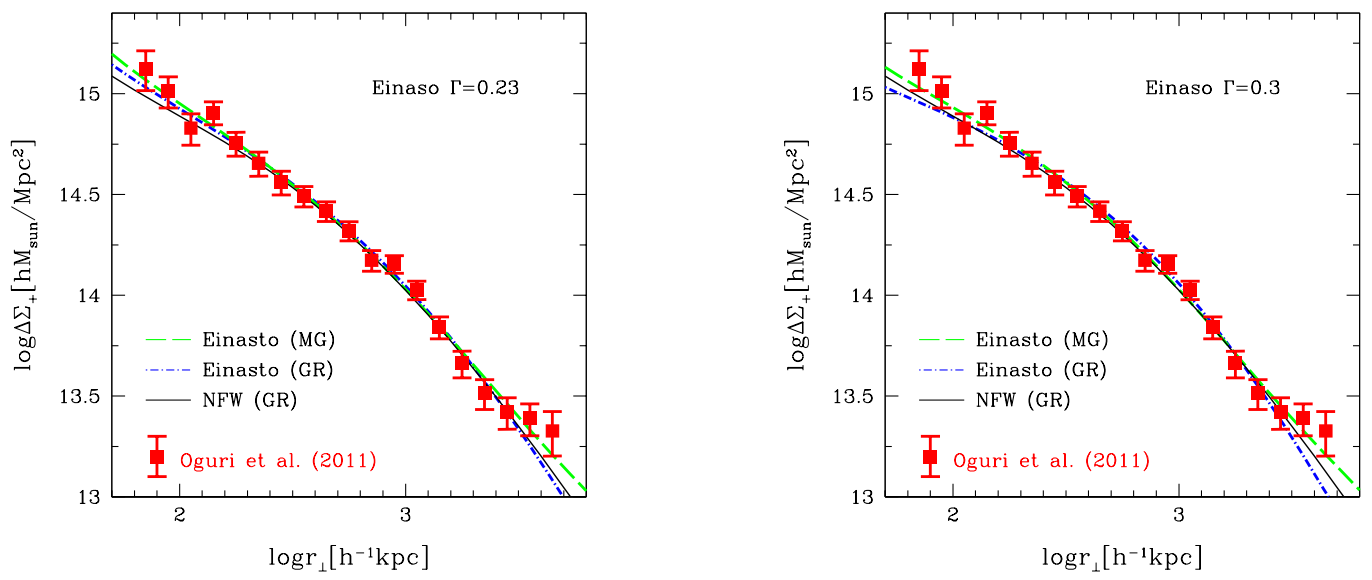

Figure 15. Same figure as figure 14, but for the differential surface mass density $\Delta \Sigma_{+}\left(r_{\perp}\right)$. The best-fit parameter for the modified gravity is $\mu=5.0$ and $\epsilon=1.8(\mu=5.0$ and $\epsilon=1.1)$ in the left (right) panel, respectively.

The generalized galileon model we considered is characterized by the two parameters, $\mu$ and $\epsilon$, which describe the amplitude and the typical scale of the modification of gravity of the Vainshtein mechanism, respectively. We have investigated how the transition in the Vainshtein mechanism in the generalized galileon model appears due to a scalar degree of freedom, which leads to a modified relation between the lensing potential and the density perturbations of matter. This effect provides us with a unique opportunity for testing gravity, using observational quantities measured through the gravitational lensing.

In the present paper, we focused our investigation on the density profile of cluster halo observed with gravitational lensing. Utilizing the observational data of the surface mass density in [76] and the differential surface mass density in [77], we obtained the constraint on the parameters $\mu$ and $\epsilon$ for the first time, assuming the NFW profile, the gNFW profile and the Einasto profile as the halo density profile. We obtained the constraint, for instance, $-0.4 \lesssim \mu \lesssim 0.6$ for $\epsilon=0.1$, and $-1.5 \lesssim \mu \lesssim 2.5$ for $\epsilon=1.0$, respectively, at 1-sigma confidence level, assuming the NFW profile (see figure 11). The constraint on $\mu$ is better for small $\epsilon$, but the constraint on $\mu$ becomes rather weak as $\epsilon$ becomes larger. Newtonian gravity is favored when the NFW profile is assumed. This result is not altered when the gNFW profile is assumed (see section 4). However, the modified gravity is favored than Newtonian gravity when the Einasto profile with large $\Gamma$ is assumed (see section 5). The constraint obtained with our method is not very tight. The original galileon model is not excluded in our test. But this method provides us with a unique chance to test the gravity theory on halo scales with cluster surveys, such as Subaru Hyper Suprime-Cam survey [98], CLASH [100], LoCuSS [101], and XXM-XXL [102].

In the present paper, we assumed the well-known density profiles of halo, which should be carefully tested with N-body simulations. In reference [46], properties of halos in the DGP modified gravity model are investigated. The DGP model possesses the Vainshtein mechanism similar to the galileon model. ${ }^{3}$ From the N-body simulations in reference [46], it is suggested that the NFW profile describes the density profile of halos in the DGP model

\footnotetext{
${ }^{3}$ In the DGP model the relation connecting the lensing potential and the density perturbation is not altered, therefore it is difficult to obtain a constraint with the method in the present paper.
} 
though the parameters of the profiles are scaled. This supports our assumption. However, in order to make the constraint robust, we need to check the modeling of the halo profile in future. As we considered the simple halo model in our analysis, our modeling of halo is valid only within the radius smaller than a few Mpc. In order to use the observational data including larger radius [94], we need a more sophisticated formulation, which is also left as a future problem.

\section{Acknowledgments}

We thank Keiichi Umetsu and Masamune Oguri for providing us with their data and useful comments. We thank Rampei Kimura and Gen Nakamura for useful discussions. We thank Jun'ichi Yokoyama and his collaborators for many useful discussions. We thank Claudia de Rham and Alister W. Graham for useful comments. We also thank Vera Richter and Chris Knobler for useful comments and supports. This work was supported by Japan Society for Promotion of Science (JSPS) Grants-in-Aid for Scientific Research (Nos. 21540270, 21244033) and JSPS Core-to-Core Program "International Research Network for Dark Energy". T.N. was supported in part by a Grant-in-Aid for JSPS Fellows.

\section{A The coefficients in the perturbation equations}

In this appendix, we present the general expressions of the coefficients in the perturbation equations in a subclass of general second-order scalar-tensor theory with the action (2.1). Following [36], we introduce

$$
\begin{aligned}
A_{0} & =\frac{\dot{\Theta}}{H^{2}}+\frac{\Theta}{H}-F-2 \frac{F_{\phi} \dot{\phi}}{H}-\frac{\mathcal{E}+\mathcal{P}}{2 H^{2}}, \\
A_{1} & =\frac{F_{\phi} \dot{\phi}}{H} \\
A_{2} & =F-\frac{\Theta}{H}, \\
B_{0} & =\frac{\dot{\phi}^{3} G_{X}}{2 H}
\end{aligned}
$$

where

$$
\begin{aligned}
\mathcal{E} & =2 X K_{X}-K+6 H X \dot{\phi} G_{X}-2 X G_{\phi}-3 H^{2} F-3 H \dot{\phi} F_{\phi}, \\
\mathcal{P} & =K-2 X\left(G_{\phi}+\ddot{\phi} G_{X}\right)+\left(3 H^{2}+2 \dot{H}\right) F+(\ddot{\phi}+2 H \dot{\phi}) F_{\phi}+2 X F_{\phi \phi}, \\
\Theta & =-X \dot{\phi} G_{X}+H F+\dot{\phi} F_{\phi} / 2,
\end{aligned}
$$

and $F_{\phi}=d F(\phi) / d \phi, G_{X}=\partial G / \partial X$ and $G_{\phi}=\partial G / \partial \phi$. Finally, we have the following expressions for the coefficients in section 2 ,

$$
\begin{aligned}
& \alpha=\frac{F_{\phi}}{F} \phi, \quad \xi=\frac{2 X G_{X}-F_{\phi}}{2 F} \phi, \quad \zeta=\frac{2\left(A_{1}+A_{2}\right) H}{\beta \dot{\phi} \phi}, \quad \lambda^{2}=\frac{B_{0} H \phi}{\beta X \dot{\phi}} \\
& \beta=-\left(A_{0}+A_{2} \frac{F_{\phi} \dot{\phi}}{F H}+\left(A_{1}+A_{2}\right) \frac{A_{2}}{F}\right) \frac{2 H^{2}}{\dot{\phi}^{2}} .
\end{aligned}
$$




\section{B Galileon model}

We consider the galileon model in curved spacetime with the action [29],

$$
S=\int d^{4} x \sqrt{-g}\left[\frac{M_{\mathrm{Pl}}^{2}}{2} R-X-\frac{r_{c}^{2}}{M_{\mathrm{Pl}}} X \square \phi+\mathcal{L}_{\mathrm{m}}\right]
$$

which is obtained by taking $F(\phi)=M_{\mathrm{Pl}}^{2}, K(X)=-X$ and $G(X)=\left(r_{c}^{2} / M_{\mathrm{Pl}}\right) X$, where $r_{c}$ is the parameter. This model admits a late-time de-Sitter attractor in a flat FRW universe. The modified Friedmann equation on the attractor can be written as

$$
\left(\frac{H(a)}{H_{0}}\right)^{2}=\frac{1}{2}\left[\Omega_{0} a^{-3}+\sqrt{\left(\Omega_{0} a^{-3}\right)^{2}+4\left(1-\Omega_{0}\right)}\right] .
$$

The parameter $r_{c}$ is related to the cosmological parameters by $r_{c}=1 /\left(54\left(1-\Omega_{0}\right)\right)^{1 / 4} H_{0}^{-1}$. In this galileon model, $\alpha, \xi, \zeta, \lambda^{2}$ and $\beta$ in (A.3) are given by the background expansion history, as follows,

$$
\begin{aligned}
& \alpha=0, \quad \xi=M_{\mathrm{Pl}}^{-2} X \phi G_{X}, \quad \zeta=\frac{2 X G_{X}}{\beta \phi}, \quad \lambda^{2}=\frac{\phi G_{X}}{\beta}, \\
& \beta=-1+2 G_{X}(\ddot{\phi}+2 H \dot{\phi})-2 M_{\mathrm{Pl}}^{-2} X^{2} G_{X}^{2} .
\end{aligned}
$$

For this galileon model along the attractor solution, it is useful to rewrite the combinations $\xi \zeta$ and $\lambda^{2} \zeta$ in terms of the matter density parameter $\Omega_{m}=\rho_{m}(a) / 3 M_{\mathrm{Pl}}^{2} H^{2}(a)[33,54]$,

$$
\xi \zeta=\frac{\left(1-\Omega_{m}\right)\left(2-\Omega_{m}\right)}{\Omega_{m}\left(5-\Omega_{m}\right)}, \quad \lambda^{2} \zeta=\left(\frac{2-\Omega_{m}}{H \Omega_{m}\left(5-\Omega_{m}\right)}\right)^{2} .
$$

In this model, we have $\mu=0.26$ and $\epsilon=0.53(\mu=0.19$ and $\epsilon=0.43)$ at $z=0.32(z=0.47)$, which corresponds to the mean redshift of the clusters to measure the surface mass density by Umetsu et al. in [75, 76] (the differential surface mass density by Oguri et al. in [77]), respectively.

\section{References}

[1] A. G. Riess et al., Observational Evidence from Supernovae for an Accelerating Universe and a Cosmological Constant, Astron. J. 116 (1998) 1009 astro-ph/arXiv:9805201

[2] S. Perlmutter et al., Measurements of Omega and Lambda from 42 High-Redshift Supernovae, Astropys. J. 517 (1999) 565 arXiv:astro-ph/9812133

[3] R. Durrer and R. Maartens, Dark energy and modified gravity arXiv:0811.4132

[4] B. Jain and J. Khoury, Cosmological tests of gravity, Annals of Physics 325 (2010) 1479 arXiv:1004.3294

[5] S. Tsujikawa, Modified gravity models of dark energy, Lect. Notes Phys 800 (2010) 99 arXiv:1101.0191

[6] E. J. Copeland, M. Sami and S. Tsujikawa, Dynamics of dark energy, Int. J. Mod. Phys. D 15 (2006) 1753 arXiv:hep-th/0603057

[7] L. Amendola and S. Tsujikawa, Dark Energy: Theory and Observations (Cambridge University Press, 2010) 
[8] W. Hu and I. Sawicki, Models of $f(R)$ cosmic acceleration that evade solar-system tests, Phys. Rev. D 76 (2007) 064004 arXiv:0705.1158

[9] S. A. Appleby and R. A. Battye, Do consistent $F(R)$ models mimic general relativity plus $\Lambda$ ?, Phys. Lett. B 654 (2007) 7 arXiv:0705.3199

[10] A. A. Starobinsky, Disappearing cosmological constant in $f(R)$ gravity, JETP Lett. 86 (2007) 157 arXiv:0706.2041

[11] S. Nojiri and S. D. Odintsov, Modified $f(R)$ gravity unifying $R^{m}$ inflation with the $\Lambda C D M$ epoch, Phys. Rev. D 77 (2008) 026007 arXiv:0710.1738

[12] A. De Felice and S. Tsujikawa, $f(R)$ theories, Living Rev. Rel. 13 (2010) 3 arXiv:1002.4928

[13] G. R. Dvali, G. Gabadadze and M. Porrati, $4 D$ gravity on a brane in 5D Minkowski space, Phys. Lett. B 485 (2000) 208 arXiv:hep-th/0005016

[14] A. Nicolis, R. Rattazzi and E. Trincherini, Galileon as a local modification of gravity, Phys. Rev. D 79 (2009) 064036 arXiv:0811.2197

[15] C. Deffayet, Cosmology on a brane in Minkowski bulk, Phys. Lett. B 502 (2001) 199 arXiv:hep-th/0010186

[16] C. Deffayet, G. R. Dvali and G. Gabadadze, Accelerated universe from gravity leaking to extra dimensions, Phys. Rev. D 65 (2002) 044023 arXiv:astro-ph/0105068

[17] A. Nicolis and R. Rattazzi, Classical and Quantum Consistency of the DGP Model, JHEP 06 (2004) 059 arXiv:hep-th/0404159

[18] D. Goubnov, K. Koyama and S. Sibiryakov, More on ghosts in the Dvali-Gabadaze-Porrati model, Phys. Rev. D 73 (2006) 044016 arXiv:hep-th/0512097

[19] M. Fairbairn and A. Goobaar, Supernova limits on brane world cosmology, Phys. Lett. B 642 (2006) 432 arXiv:astro-ph/0511029

[20] R. Maartens and E. Majerotto, Observational constraints on self-accelerating cosmology, Phys. Rev. D 74 (2006) 023004 arXiv:astro-ph/0603353

[21] Y. S. Song, I. Sawicki and W. Hu, Large-Scale Tests of the DGP Model, Phys. Rev. D 75 (2007) 064003 arXiv:astro-ph/0606286

[22] M. A. Luty, M. Porrati and R. Rattazzi, Strong interactions and stability in the DGP model, JHEP 09 (2003) 029 arXiv:hep-th/0303116

[23] C. Deffayet, G. Esposito-Farese and A. Vikman, Covariant Galileon, Phys. Rev. D 79 (2009) 084003 arXiv:0901.1314

[24] N. Chow and J. Khoury, Galileon cosmology, Phys. Rev. D 80 (2009) 024037 arXiv:0905.1325

[25] F. P. Silva and K. Koyama, Self-accelerating universe in Galileon cosmology, Phys. Rev. D 80 (2009) 121301 arXiv:0909.4538

[26] T. Kobayashi, H. Tashiro and D. Suzuki, Evolution of linear cosmological perturbations and its observational implications in Galileon-type modified gravity, Phys. Rev. D 81 (2010) 063513 arXiv:0912.4641

[27] T. Kobayashi, Cosmic expansion and growth histories in Galileon scalar-tensor models of dark energy, Phys. Rev. D 81 (2010) 103533 arXiv:1003.3281

[28] A. De Felice and S. Tsujikawa, Cosmology of a Covariant Galileon Field, Phys. Rev. Lett. 105 (2010) 111301 arXiv:1007.2700

[29] C. Deffayet, O. Pujolas, I. Sawicki and A. Vikman, Imperfect dark energy from kinetic gravity braiding, JCAP 10 (2010) 026 arXiv:1008.0048

[30] T. Kobayashi, M. Yamaguchi and J. Yokoyama, Inflation Driven by the Galileon Field, Phys. 
Rev. Lett. 105 (2010) 231302 arXiv:1008.0603

[31] A. De Felice and S. Tsujikawa, Generalized Galileon cosmology, Phys. Rev. D 12 (2011) 124029 arXiv: 1008.4236

[32] S. Nesseris, A. De Felice and S. Tsujikawa, Observational constraints on Galileon cosmology, Phys. Rev. D 82 (2010) 124054 arXiv:1010.0407

[33] R. Kimura and K. Yamamoto, Large scale structures in the kinetic gravity braiding model that can be unbraided, JCAP 04 (2011) 025 arXiv:1011.2006

[34] G. W. Horndeski, Second-order scalar-tensor field equations in a four-dimensional space, Int. J. Theor. Phys. 10 (1974) 363

[35] C. Deffayet, X. Gao, D. A. Steer, and G. Zahariada, From k-essence to generalized Galileons, Phys. Rev. D 80 (2011) 064039 arXiv:1103.3260

[36] T. Kobayashi, M. Yamaguchi and J. Yokoyama, Generalized G-Inflation - Inflation with the most general second-order field equations -, Prog. Theor. Phys. 126 (2011) 3 arXiv:1105.5723

[37] C. M. Will, The Confrontation between General Relativity and Experiment, Living Rev. Relativity 9 (2006) 3 arXiv:gr-qc/0510072

[38] D. F. Mota and J. D. Barrow, Varying Alpha in a More Realistic Universe, Phys. Lett. B 581 (2004) 141 arXiv:astro-ph/0306047

[39] J. Khoury and A. Weltman, Chameleon Fields: Awaiting Surprises for Tests of Gravity in Space, Phys. Rev. Lett. 93 (2004) 171104 arXiv:astro-ph/0309300

[40] J. Khoury and A. Weltman, Chameleon cosmology, Phys. Rev. D 69 (2004) 044026 arXiv:astro-ph/0309411

[41] K. Hinterbichler and J. Khoury, Screening Long-Range Forces through Local Symmetry Restoration, Phys. Rev. Lett. 104 (2010) 231301 arXiv:1001.4525

[42] K. Hinterbichler, J. Khoury, A. Levy and A. Matas, Symmetron cosmology, Phys. Rev. D 84 (2011) 103521 arXiv:1107.2112

[43] A. I. Vainshtein, To the problem of nonvanishing gravitation mass, Phys. Lett. B 39 (1972) 393

[44] R. Kimura, T. Kobayashi and K. Yamamoto, Vainshtein screening in a cosmological background in the most general second-order scalar-tensor theory, Phys. Rev. D 85 (2012) 024023 arXiv:1111.6749

[45] L. Hui and A. Nicolis, An observational test of the Vainshtein mechanism, arXiv:1201.1508

[46] F. Schmidt, Dynamical masses in the modified gravity, Phys. Rev. D 81 (2010) 103002 arXiv:1003.0409

[47] P. Chang and L. Hui, Stellar Structure and Tests of Modified Gravity, Astrophys. J. 732 (2011) 25 arXiv:1011.4107

[48] M. Wyman, Galilean-invariant scalar fields can strengthen gravitational lensing, Phys. Rev. Lett. 106 (2011) 201102 arXiv:1101.1295

[49] A.-C. Davis, E. A. Lim, J. Sakstein and D. Shaw, Modified Gravity Makes Galaxies Brighter, arXiv:1102.5278

[50] P. Burikham and S. Panpanich, Effects of chameleon scalar field on rotation curves of the galaxies, arXiv:1103.1198

[51] G.-B. Zhao, B. Li and K. Koyama, Testing gravity using the environmental dependence of dark matter halos, Phys. Rev. D 107 (2011) 071303 arXiv:1105.0922

[52] B. Jain and J. VanderPlas, Tests of modified gravity with dwarf galaxies, JCAP 10 (2011) 032 arXiv:1106.0065 
[53] Y. Li and W. Hu, Chameleon halo modeling in $f(R)$ gravity, Phys. Rev. D 84 (2011) 084033 arXiv:1107.5120

[54] T. Narikawa, R. Kimura, T. Yano and K. Yamamoto, Halo models in modified gravity theories with self-accelerated expansion, IJMP D 20 (2011) 2383 arXiv:1108.2346

[55] J. Clampitt, B. Jain and J. Khoury, Halo scale predictions of symmetron modified gravity, JCAP 01 (2012) 030 arXiv:1110.2177

[56] L. Lombriser, et al., Cluster density profiles as a test of modified gravity, arXiv:1111.2020

[57] L. Hui, A. Nicolis and C. Stubbs, Equivalence Principle Implications of Modified Gravity Models, Phys. Rev. D 80 (2009) 104002 arXiv:0905.2966

[58] S. Sjors and E. Mortsell, Spherically Symmetric Solutions in Massive Gravity and Constraints from Galaxies, arXiv:1111.5961

[59] J. F. Navarro, C. S. Frenk and S. D. M. White, The structure of cold dark matter halos, Astrophys. J. 462 (1996) 563 arXiv:astro-ph/9508025

[60] J. F. Navarro, C. S. Frenk and S. D. M. White, A universal density profile from hierarchical clustering, Astrophys. J. 490 (1997) 493 arXiv:astro-ph/9611107

[61] A. De Felice, R. Kase and S. Tsujikawa, Vainshtein mechanism in second-order scalar-tensor theories, Phys. Rev. D 85 (2012) 044059 arXiv:1111.5090

[62] A. Shirata, T. Shiromizu, N. Yoshida and Y. Suto, Galaxy clustering constraints on deviations from Newtonian gravity at cosmological scales, Phys. Rev. D 71 (2005) 064030 arXiv:astro-ph/0501366

[63] A. Shirata, Y. Suto, C. Hikage, T. Shiromizu and N. Yoshida, Galaxy clustering constraints on deviations from Newtonian gravity at cosmological scales. II. Perturbative and numerical analyses of power spectrum and bispectrum, Phys. Rev. D 76 (2007) 044026 arXiv:0705.1311

[64] L. Guzzo et al., Guzzo 0802.1944 A test of the nature of cosmic acceleration using galaxy redshift distortions, Nature 451 (2008) 541arXiv:0802.1944

[65] K. Yamamoto, T. Sato, G. Huetsi, Testing general relativity with the multipole spectra of the SDSS luminous red galaxies, Prog. Theor. Phys. 120 (2008) 609 arXiv:0805.4789

[66] R. Reyes, R. Mandelbaum, U. Seljak, T. Baldauf, J. E. Gunn, L. Lombriser and R. E. Smith, Confirmation of general relativity on large scales from weak lensing and galaxy velocities, Nature 464 (2010) 256 arXiv:1003.2185

[67] K. Yamamoto, G. Nakamura, G. Huetsi, T. Narikawa and T. Sato, Constraint on the cosmological $f(R)$ model from the multipole power spectrum of the SDSS luminous red galaxy sample and prospects for a future redshift survey, Phys. Rev. D 81 (2010) 103517 arXiv:1004.3231

[68] R. Kimura, T. Kobayashi and K. Yamamoto, Observational Constraints on Kinetic Gravity Braiding from the Integrated Sachs-Wolfe Effect, arXiv:1110.3598

[69] F. Schmidt, A. Vikhlinin and W. Hu , Cluster constraints on $f(R)$ gravity, Phys. Rev. D 80 (2009) 083505 arXiv:0908.2457

[70] A. S. Bolton, S. Rappaport and S. Burles, Constraint on the post-Newtonian parameter $\gamma$ on galactic size scales, Phys. Rev. D 74 (2006) 061501 arXiv:astro-ph/0607657

[71] D. J. Kapner, et al., Tests of the Gravitational Inverse-Square Law below the Dark-Energy Length Scale, Phys. Rev. Lett. 98 (2007) 021101 arXiv:hep-ph/0611184

[72] T. Smith, Testing gravity on kiloparsec scales with strong gravitational lenses, arXiv:0907.4829

[73] J. Schwab, A. S. Bolton and S. A. Rappaport, Galaxy-scale strong-lensing tests of gravity and geometric cosmology: constraints and systematic limitations, Astrophys. J. 708 (2010) 750 
arXiv:0907.4992

[74] J.-P. Uzan, Tests of general relativity on astrophysical scales, Gen. Relativ. Gravit. 42 (2010) 2219 arXiv:0908.2243

[75] K. Umetsu, T. Broadhurst, A. Zitrin, E. Medezinski and L. Hsu, Cluster mass profiles from a Bayesian analysis of weak-lensing distortion and magnification measurements: applications to Subaru data, ApJ 729 (2011) 127 arXiv:1011.3044

[76] K. Umetsu, T. Broadhurst, A. Zitrin, E. Medezinski, D. Coe and M. Postman, A precise cluster mass profile averaged from the highest-quality lensing data, ApJ 738 (2011) 41 arXiv:1105.0444

[77] M. Oguri, et al., Combined strong and weak lensing analysis of 28 clusters from the Sloan Giant Arcs Survey, Mon. Not. R. Astron. Soc. 420 (2012) 3213 arXiv:1109.2594

[78] R. Wojtak, S. H. Hansen and J. Hjorth, Gravitational redshift of galaxies in clusters as predicted by general relativity, Nature 477 (2011) 567 arXiv:1109.6571

[79] S. Miyazaki et al. Subaru Prime Focus Camera - Suprime-Cam, Publ. Astron. Soc. Japan 54 (2002) 833 arXiv:astro-ph/0211006

[80] E. Komatsu, et al., Seven-year Wilkinson Microwave Anisotropy Probe (WMAP) Observations: Cosmological Interpretation, Astrophys. J. Suppl. 192 (2011) 18 arXiv:1001.4538

[81] M. Bartelmann and P. Schneider, Weak Gravitational lensing, Phys. Rep. 340 (2001) 291 arXiv:astro-ph/9912508

[82] S. Dodelson, Modern Cosmology, (Academic Press, 2003)

[83] B. Moore, et al., Cold collapse and the core catastrophe, Mon. Not. R. Astron. Soc. 310 (1999) 1147 arXiv:astro-ph/9903164

[84] J. F. Navarro, et al., The inner structure of $\Lambda$ CDM haloes -III. Universality and asymptotic slopes, Mon. Not. R. Astron. Soc. 349 (2004) 1039 arXiv:astro-ph/0311231

[85] D. Merritt, J. F. Navarro, A. Ludlow and A. Jenkins, A universal density profile for dark and luminous matter?, Astrophys. J. 624 (2005) L85 arXiv:astro-ph/0502515

[86] D. Merritt, A. W. Graham, B. Moore, J. Diemand and B. Terzic, Empirical models for dark matter halos. I. Nonparametric construction of density profiles and comparison with parametric models, Astron. J. 132 (2006) 2685 arXiv:astro-ph/0509417

[87] E. Hayashi and S. D. M. White, Understanding the shape of the halo-mass and galaxy-mass cross-correlation functions, Mon. Not. Roy. Astron. Soc. 388 (2008) 2 arXiv:0709.3933

[88] L. Gao, et al., The redshift dependence of the structure of massive $\Lambda$ cold dark matter haloes, Mon. Not. Roy. Astron. Soc. 387 (2008) 536 arXiv:0711.0746

[89] J. Diemand, Clumps and streams in the local dark matter distribution, Nature 454 (2008) 735 arXiv:0805.1244

[90] V. Springel et al., Prospects for detecting supersymmetric dark matter in the galactic halo, Nature 456 (2008) 73 arXiv:0809.0894

[91] V. Springel et al., The Aquarius Project: the subhaloes of galactic haloes, Mon. Not. R. Astron. Soc. 391 (2008) 1685 arXiv:0809.0898

[92] H. Zhao, Analytical models for galactic nuclei, Mon. Not. R. Astron. Soc. 278 (1996) 488 arXiv:astro-ph/9509122

[93] Y. P. Jing and Y. Suto, The density profiles of the dark matter halo are not universal, Astrophys. J. 529 (2000) L69 arXiv:astro-ph/9909478

[94] R. Mandelbaum, et al., Density profiles of galaxy groups and clusters from SDSS galaxy-galaxy weak lensing, Mon. Not. R. Astron. Soc. 372 (2006) 758 arXiv:astro-ph/0605476 
[95] J. Einasto, On the construction of a composite model for the Galaxy and on the determination of the system of Galactic parameters, Trudy Inst. Astropfiz. Alma-Ata, 5 (1965) 87

[96] A. W. Graham, et al., Empirical models for Dark Matter Halos. II. Inner Profile Slopes, Dynamical Profiles, and $\rho / \sigma^{3}$, Astron. J. 132 (2006) 2701 arXiv:astro-ph/0608613

[97] J. F. Navarro, et al., The diversity and similarity of simulated cold dark matter haloes, Mon. Not. Roy. Astron. Soc. 402 (2010) 21 arXiv:0810.1522

[98] M. Takada, Subaru Hyper Suprime-Cam Project, American Institute of Physics Conference Series 1279 (2010) 120

[99] A. D. Ludlow, et al., The density and pseudo-phase-space density profiles of cold dark matter haloes, Mon. Not. R. Astron. Soc. 415 (2011) 3895 arXiv:1102.0002

[100] M. Postman, et al., Cluster Lensing And Supernova Survey with Hubble: An Overview, Astrophys. J. Suppl. 199 (2012) 25 arXiv:1106.3328

[101] N. Okabe, M. Takada, K. Umetsu, T. Futamase, G. P. Smith, LoCuSS: Subaru Weak Lensing Study of 30 Galaxy Clusters, Publ. Astron. Soc. Japan 62 (2010) 811 arXiv:0903.1103

[102] M. Pierre, F. Pacaud, J. B. Juin, J. B. Melin, P. Valageas, N. Clerc and P. S. Corasaniti, Precision cosmology with a wide area XMM cluster survey, Mon. Not. R. Astron. Soc. 414 (2011) 1732 arXiv:1009.3182 